\title{
The Rost invariant has trivial kernel for quasi-split groups of low rank
}

Ryan Skip Garibaldi

\begin{abstract}
For $G$ a simple simply connected algebraic group defined over a field $F$, Rost has shown that there exists a canonical map $R_{G}: H^{1}(F, G) \rightarrow H^{3}(F, \mathbb{Q} / \mathbb{Z}(2))$. This includes the Arason invariant for quadratic forms and Rost's mod 3 invariant for exceptional Jordan algebras as special cases. We show that $R_{G}$ has trivial kernel if $G$ is quasi-split of type $E_{6}$ or $E_{7}$. A case-by-case analysis shows that it has trivial kernel whenever $G$ is quasi-split of low rank.
\end{abstract}

Mathematics Subject Classification (2000). 20G10 (17B25).

Keywords. Rost invariant, exceptional groups.

For $G$ a simple simply connected algebraic group over a field $F$, the set of all natural transformations of functors

$$
H^{1}(?, G) \longrightarrow H^{3}(?, \mathbb{Q} / \mathbb{Z}(2))
$$

is a finite cyclic group [KMRT98, §31] with a canonical generator. (Here $H^{i}(?, M)$ is the Galois cohomology functor which takes a field extension of the base field $F$ and returns a group if $M$ is abelian and a pointed set otherwise. When $F$ has characteristic $0, \mathbb{Q} / \mathbb{Z}(2)$ is defined to be $\lim _{\longrightarrow} \boldsymbol{\mu}_{n}^{\otimes 2}$ for $\boldsymbol{\mu}_{n}$ the algebraic groups of $n$th roots of unity; see [EKLV98, p. 95] or [Gil00, I.1(b)] for a more complete definition.) This generator is called the Rost invariant of $G$ and we denote it by $R_{G}$. In an abuse of notation, we also write $R_{G}$ for the map $H^{1}(F, G) \longrightarrow H^{1}(F, \mathbb{Q} / \mathbb{Z}(2))$.

This map provides a useful invariant for algebraic structures classified by $H^{1}(F, G)$, and an important and typically difficult question is to describe the kernel of $R_{G}$. For example, when $G$ is split of type $D_{n}, R_{G}$ is essentially the Arason invariant $I^{3} F \rightarrow H^{3}(F, \mathbb{Z} / 2)$ for quadratic forms, where $I^{n} F$ is as usual the $n$th power of the ideal $I F$ of even-dimensional quadratic forms in the Witt ring of $F$. That the kernel of the Arason invariant is precisely $I^{4} F$ is a quite difficult result due independently to Merkurjev-Suslin [MS91] and Rost. (The proof of the main result of this paper somehow boils down to this one fact.) In general, one doesn't even know if the kernel of $R_{G}$ is trivial. On the other hand, the question becomes tractable if we assume that $G$ is quasi-split. Generally $R_{G}$ has nontrivial kernel; we give easy examples where $G$ is split of type $D_{8}$ (in 1.9) and $B_{7}$ (in 1.6), and quasi-split of type ${ }^{2} A_{6}$ (in 1.11). It should be mentioned that $R_{G}$ can 
have nontrivial kernel when $G$ is split of type $E_{8}$ as well; Gille [Gil, Appendix] has produced an example by applying his results from [Gil00] to reduce the question to the same one for a split group of type $D_{8}$.

The principal result in this paper is to enlarge the list of quasi-split groups for which the Rost invariant is known to have trivial kernel.

Main Theorem 0.1. Suppose that $G$ is a quasi-split simply connected group of type $E_{6}$ or $E_{7}$. Then the Rost invariant $R_{G}$ has trivial kernel.

0.2. There are some easy consequences of this theorem that may help the reader place it in context. The first is that as a vastly less powerful corollary, we obtain Serre's "Conjecture II" for quasi-split groups of type $E_{6}$ and $E_{7}$, in that if $F$ has $p$-cohomological dimension $\leq 2$ for $p=2,3$ (see [Ser94, I.3] for a definition), then the main theorem implies that $H^{1}(F, G)$ is trivial. This conjecture appeared in print back in 1962 [Ser62], and remained open for such groups until the 1990s, when Chernousov (unpublished) and Gille [Gil01] proved it (amongst other cases) independently and by different methods. Here we get it for free from the Main Theorem.

0.3. Another consequence is the following: Suppose that $L$ is a field extension of $F$ of degree relatively prime to 2 and 3 and that $G$ is a group of type $E_{6}$ or $E_{7}$. Serre asked in [Ser95, p. 233, Q. 1] if the natural map $H^{1}(F, G) \rightarrow H^{1}(L, G)$ is injective. Our Main Theorem gives the partial answer that it has trivial kernel in the case where $G$ is quasi-split. This result was already known by experts in the area using arguments special to groups of type $E_{6}$ and $E_{7}$, but as for Conjecture II we get it for free here.

0.4. There is also an application to finite-dimensional algebras. There is a large family of nonassociative algebras with involution called structurable algebras which includes central simple associative algebras with involution (as studied in [KMRT98]) and Jordan algebras (with involution the identity), see [All94] for a survey. The simple structurable algebras have all been classified, and they consist (roughly) of the two families already mentioned plus four others. The most poorly understood of these four additional types consists of 56-dimensional algebras all of which are isomorphic over a separably closed field and have automorphism group which is simply connected of type $E_{6}$. Call algebras belonging to this class Brown algebras. There is a natural equivalence relation defined on the set of structurable algebras called isotopy [AH81] which is weaker than isomorphism, and in the case of Jordan algebras is the same as the traditional notion of isotopy. For Albert algebras, it is known that any algebra isotopic to the split one is actually split. (This is equivalent to the cohomological statement that the map $H^{1}\left(F, F_{4}\right) \rightarrow H^{1}\left(F, E_{6}\right)$ induced by the embedding $F_{4} \rightarrow E_{6}$ described in 2.4 has trivial kernel.) The Main Theorem here combined with [Gar01b, 4.16(2), 5.12] shows that an analogous conclusion holds for Brown algebras, i.e., a Brown algebra isotopic to the split one is 
quasi-split. This was previously unknown. (This has the cohomological interpretation that the map $H^{1}\left(F, E_{6}^{K}\right) \rightarrow H^{1}\left(F, E_{7}\right)$ induced by the embedding $E_{6}^{K} \rightarrow E_{7}$ described in 3.5 has trivial kernel.)

The material in [KMRT98] is sufficient to show that the kernel of the Rost invariant is trivial for quasi-split groups of type $G_{2}, D_{4}$ (including those of trialitarian type [KMRT98, 40.16]), and $F_{4}$, at least away from the "bad primes" 2 and 3. As easy corollaries to results needed for the $E_{6}$ and $E_{7}$ cases, we get analogous results for groups of type ${ }^{2} A_{n}, B_{n}$, and nontrialitarian groups of type $D_{n}$ with small $n$ in Section 1. Since $H^{1}(F, G)$ is always trivial for $G$ split of type $A_{n}$ or $C_{n}$, we get the following:

Theorem 0.5. Suppose that $G$ is a simple simply connected algebraic group. If $G$ is

- quasi-split of (absolute) rank $\leq 5$;

- quasi-split of type $B_{6}, D_{6}$, or $E_{6}$; or

- split of type $D_{7}$ or $E_{7}$,

then the Rost invariant $R_{G}$ has trivial kernel.

The proofs of these theorems that we will give here and the material in [KMRT98] rely on the ground field having "good" characteristic, meaning for our purposes $\neq 2,3$. However, it is a consequence of Gille's main theorem in [Gil00] that one only needs to prove that the Rost invariant has trivial kernel for fields of characteristic 0. Consequently, all fields considered here will be assumed to have characteristic $\neq 2,3$, but our two theorems will still hold for all characteristics. (Of course, in prime characteristic the group $\mathbb{Q} / \mathbb{Z}(2)$ must be defined somewhat differently [Gil00], but this affects neither the statement of the theorems nor our proofs.)

Section 1 dispenses with the classical groups. (Some of that material is useful later.) Sections 2 and 3 contain the material necessary to reduce questions about the Rost invariant for a larger group to a subgroup. That material easily reduces the proof of the main theorem to considering the quasi-split ${ }^{2} E_{6}$ case, which is treated in the remaining Sections 4 through $7 .^{1}$

Remark 0.6 (Noninjectivity for $F_{4}$ ). We caution the reader that even when the Rost invariant has trivial kernel, it may be far from injective. For example, for $F_{4}$ the split group of type $F_{4}$, the set $H^{1}\left(F, F_{4}\right)$ classifies Albert $F$-algebras. From known facts about Albert algebras, it is easy to show that two classes $\alpha_{1}, \alpha_{2}$ corresponding to isotopic Jordan algebras $J_{1}, J_{2}$ have the same Rost invariant. Since there are many isotopic Albert algebras which are not isomorphic (for example,

\footnotetext{
${ }^{1}$ After this paper was released as a preprint, Chernousov sent to me a different proof of the ${ }^{2} E_{6}$ case [Che00], which uses a completely different argument. His proof will be published elsewhere.
} 
over $\mathbb{R}$ there are 3 isomorphism classes of Albert algebras and two of these are isotopic [Jac71, p. 119]), the Rost invariant for $F_{4}$ has trivial kernel but is typically not injective.

\section{Notations and conventions}

All algebraic groups considered here will be affine. We say that an algebraic group $G$ is simple if it has finite center and no noncentral closed normal subgroups defined over an algebraic closure. When we say that a group is "of type $T_{n}$ ", we implicitly mean that it is simple of that type. We will use the standard notations $\mathbb{G}_{m}, \mathbb{G}_{a}$, and $\boldsymbol{\mu}_{n}$ for the algebraic groups with $F$-points $F^{*}, F$, and the $n$th roots of unity in $F$, and $G^{\circ}$ will always denote the identity component of an algebraic group $G$. For a variety $X$ we write $X(F)$ for its $F$-points.

Our notation for quadratic forms will follow the standard reference [Lam73], with two quirks: We use the Pfister-approved notation for Pfister forms, so $\ll a_{1}, \ldots, a_{n} \gg:=\left\langle 1,-a_{1}\right\rangle \otimes \cdots \otimes\left\langle 1,-a_{n}\right\rangle$, and we write $\mathcal{H}$ for the hyperbolic plane $\langle 1,-1\rangle$.

The standard reference for Galois cohomology is [Ser94, §I.5], and for algebras with involution (including the groups $\operatorname{Spin}(A, \sigma), O(A, \sigma)$, and $S O(A, \sigma))$ it is [KMRT98].

\section{Quasi-split groups of type $A, B$, and $D$}

As indicated in the introduction, the Rost invariant "should" have trivial kernel for quasi-split groups of small rank. To prove this for $E_{6}$, we will need a result on groups of type $D$, which also easily settles this question for groups of type $A$ and $B$. (For the results in this section, our global hypothesis that our fields have characteristic $\neq 3$ is not required; we need only assume characteristic $\neq 2$.) For $q$ a nondegenerate quadratic form over $F$, there is a short exact sequence of algebraic groups

$$
1 \longrightarrow C \longrightarrow \operatorname{Spin}(q) \longrightarrow S O(q) \longrightarrow 1
$$

with $C$ isomorphic to $\boldsymbol{\mu}_{2}$.

Lemma 1.2. For $q$ a d-dimensional nondegenerate quadratic form with anisotropic part of dimension $d_{\mathrm{an}}$ such that $d \geq 5$ and $d+d_{\mathrm{an}}<16$, the kernel of the Rost invariant of $\operatorname{Spin}(q)$ is precisely the image of $H^{1}(F, C)$ in $H^{1}(F, \operatorname{Spin}(q))$.

The hypothesis $d \geq 5$ ensures that $\operatorname{Spin}(q)$ is simple and simply connected, so that it makes sense to speak of the Rost invariant $R_{\operatorname{Spin}(q)}$.

Proof. The set $H^{1}(F, S O(q))$ classifies quadratic forms of the same dimension and 
discriminant as $q$ [KMRT98, 29.29]. For $\alpha \in H^{1}(F, \operatorname{Spin}(q))$ we set $q_{\alpha}$ to be the quadratic form corresponding to the image of $\alpha$ in $H^{1}(F, S O(q))$. Then $q_{\alpha}-q$ is not only even-dimensional with trivial discriminant (i.e., $q_{\alpha}-q \in I^{2} F$ ), but since $q_{\alpha}$ comes from $H^{1}(F, \operatorname{Spin}(q))$, it has the same Clifford invariant as $q$ [KMRT98, 31.11] and so $q_{\alpha}-q \in I^{3} F$ by Merkurjev's Theorem. As described in [KMRT98, p. 437], the Rost invariant of $\alpha$ is the Arason invariant $e_{3}\left(q_{\alpha}-q\right) \in H^{3}(F, \mathbb{Z} / 2)$. (Since $\mathbb{Z} / 2=\boldsymbol{\mu}_{2}^{\otimes 2}$, we can consider $\mathbb{Z} / 2$ to be a subgroup of $\mathbb{Q} / \mathbb{Z}(2)$ and hence $H^{3}(F, \mathbb{Z} / 2)$ is a subgroup of $H^{3}(F, \mathbb{Q} / \mathbb{Z}(2))$.)

Suppose first that $\alpha$ is in the image of $H^{1}(F, C)$. Sequence (1.1) induces an exact sequence

$$
S O(q)(F) \longrightarrow H^{1}(F, C) \longrightarrow H^{1}(F, \operatorname{Spin}(q)) \longrightarrow H^{1}(F, S O(q)),
$$

and since the Rost invariant $R_{\operatorname{Spin}(q)}$ "factors through" $H^{1}(F, S O(q))$, certainly $R_{\operatorname{Spin}(q)}(\alpha)$ is trivial.

Conversely, suppose that $\alpha$ is in the kernel of the Rost invariant. Then $e_{3}\left(q_{\alpha}-q\right)$ is trivial, but as mentioned in the introduction the kernel of $e_{3}$ is precisely $I^{4} F$. Since $\operatorname{dim} q_{\alpha}=\operatorname{dim} q=d$, the hypotheses on $q$ ensure that the dimension of the anisotropic part of $q_{\alpha}-q$ is strictly less than 16 . Since $q_{\alpha}-q \in I^{4} F$, it is hyperbolic by the Arason-Pfister Hauptsatz [Lam73, X.3.1]. Thus $q_{\alpha}$ is isomorphic to $q$ and $\alpha$ is in the kernel of the map $H^{1}(F, \operatorname{Spin}(q)) \rightarrow H^{1}(F, S O(q))$, which is just the image of $H^{1}(F, C)$.

The first map in (1.3) is the spinor norm, which immediately produces the following lemma.

Corollary 1.4. Suppose that $q$ is as in Lemma 1.2. Then the kernel of the Rost invariant is isomorphic to $F^{*} / S N(q) F^{* 2}$, where $S N(q)$ is the image of the spinor norm map $S O(q)(F) \rightarrow F^{*} / F^{* 2}$.

1.5. Quasi-split simply connected groups of type $B_{n}$ are actually split, so of the form $\operatorname{Spin}(q)$ for $q=n \mathcal{H} \perp\langle 1\rangle$. In terms of the lemma, $d=2 n+1$ and $d_{\text {an }}=1$. So $q$ satisfies the hypotheses for $2 \leq n \leq 6$. Since $q$ is isotropic, it has surjective spinor norm, so the Rost invariant for a split group of type $B_{n}$ has trivial kernel for $2 \leq n \leq 6$.

Example 1.6 $\left(B_{7}\right)$. As just mentioned, the split simply connected group of type $B_{7}$ is isomorphic to $\operatorname{Spin}(q)$ for $q=7 \mathcal{H} \perp\langle 1\rangle$. The Rost invariant $R_{\operatorname{Spin}(q)}$ can have nontrivial kernel. Sequence (1.1) induces an exact sequence

$$
H^{1}(F, \operatorname{Spin}(q)) \longrightarrow H^{1}(F, S O(q)) \stackrel{\partial}{\longrightarrow} H^{2}\left(F, \boldsymbol{\mu}_{2}\right)
$$

where the set $H^{1}(F, S O(q))$ classifies nondegenerate quadratic forms with the same dimension (15) and discriminant $\left(1 \cdot F^{* 2}\right)$ as $q$.

Fix a base field $F$ and a nonhyperbolic 4 -fold Pfister form $\varphi$ over $F$ (e.g. $F=\mathbb{R}$, 
$\varphi=\ll-1,-1,-1,-1 \gg)$. Set $q_{\alpha}=-\varphi^{\prime}$ for $\varphi^{\prime}$ such that $\varphi=\langle 1\rangle \perp \varphi^{\prime}$. Then $\operatorname{disc} q_{\alpha}=(-1)^{\left(\begin{array}{c}15 \\ 2\end{array}\right)} \operatorname{det}\left(-\varphi^{\prime}\right)=1 \cdot F^{* 2}$, so there is a unique element of $H^{1}(F, S O(q))$ corresponding to $q_{\alpha}$. The image of $q_{\alpha}$ under the connecting homomorphism $\partial$ is $\left[C_{0}\left(q_{\alpha}-q\right)\right]$, which by [Lam73, V.2.10] is the same as $\left[C\left(q_{\alpha}-q\right)\right]$ which is trivial since $q_{\alpha}-q=-\varphi \in I^{3} F$. Thus $q_{\alpha}$ is the image of some $\alpha$ in $H^{1}(F, \operatorname{Spin}(q))$. But then $R_{\operatorname{Spin}(q)}(\alpha)=e_{3}\left(q_{\alpha}-q\right)=e_{3}(-\varphi)$, which is trivial since $\varphi \in I^{4} F$.

1.8. An analysis for groups of type $D_{n}$ similar to the one in 1.5 for $B_{n}$ shows that the Rost invariant for a simply connected group is trivial for groups of type ${ }^{1} D_{n}$ with $3 \leq n \leq 7$ and for groups of type ${ }^{2} D_{n}$ with $3 \leq n \leq 6$. As in the $B$ case, we show that one of these bounds is sharp.

Example $1.9\left({ }^{1} D_{8}\right)$. The situation here is quite similar to the one in Example 1.6, except that $q=8 \mathcal{H}$. Use the same base field $F$ and nonsplit 4 -fold Pfister form $\varphi$ from before. There is a unique element of $H^{1}(F, S O(q))$ corresponding to $\varphi$ and since $\varphi=\varphi-q \in I^{4} F$, the same reasoning shows that there is a nontrivial class in $H^{1}(F, \operatorname{Spin}(q))$ which is the inverse image of $\varphi$ and which has trivial Rost invariant.

Lemma 1.2 easily deals with quasi-split groups of type ${ }^{2} A_{n}$ of low rank.

Corollary 1.10. If $G$ is a quasi-split simply connected group of type ${ }^{2} A_{n}$ with $n \leq 5$, the kernel of the Rost invariant $R_{G}$ is trivial.

Proof. Set $K$ to be the quadratic field extension of $F$ which splits $G$ and take $\left(V, h^{d}\right)$ to be a "maximally split" $(n+1)$-dimensional hermitian form over $K$. (See below for a more explicit description.) Then $G$ is $S U\left(V, h^{d}\right)$, the algebraic group with $F$-points

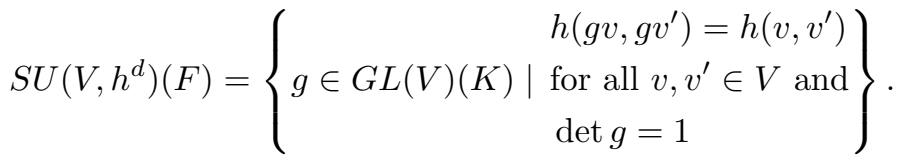

The trace form of $h^{d}$ is defined to be the quadratic form $q^{d}$ on $V$ considered as a $2(n+1)$-dimensional vector space over $F$ given by $q^{d}(v)=h^{d}(v, v)$. Then

$$
h^{d}=\left\{\begin{array}{ll}
m \mathcal{H} & \text { if } n+1=2 m, \\
m \mathcal{H} \perp\langle 1\rangle & \text { if } n+1=2 m+1
\end{array} \text { and } q^{d}= \begin{cases}2 m \mathcal{H} & \text { if } n+1=2 m, \\
2 m \mathcal{H} \perp \ll d \gg & \text { if } n+1=2 m+1,\end{cases}\right.
$$

where $K=F(\sqrt{d})$ if $n=2 m$ for some integer $m$, and the $\mathcal{H}$ occurring in the description of $h^{d}$ is the usual unitary hyperbolic plane as described in [Sch85, 7.7.3].

The set $H^{1}(F, G)$ classifies nonsingular hermitian forms $h$ on $V$ which have the same dimension and discriminant as $h^{d}$ [KMRT98, p. 403]. The group $G$ 
embeds in $S O\left(V, q^{d}\right)$ in an obvious manner. The corresponding map $H^{1}(F, G) \rightarrow$ $H^{1}\left(F, S O\left(V, q^{d}\right)\right)$ sends $h$ to its trace form $q$, and this map is an injection by [Sch85, 10.1.1(ii)]. Moreover, the Rost invariant $R_{G}(h)$ is just $e_{3}\left(q-q^{d}\right)$ by [KMRT98, 31.44]. Since $\operatorname{dim} q^{d}=2 n+2<13$ and the anisotropic part of $q^{d}$ has dimension 0 (if $n+1$ is even) and 2 (if $n+1$ is odd), as in the proof of Lemma 1.2, if $R_{G}(h)$ is trivial, $q \cong q^{d}$ and so $h \cong h^{d}$.

Example 1.11 $\left({ }^{2} A_{6}\right)$. Take $F=\mathbb{R}, K=\mathbb{C}$, and consider $G=S U\left(V, h^{d}\right)$ for $h^{d}$ the hermitian form $3 \mathcal{H} \perp\langle 1\rangle$ over $K$, so that $G$ is simply connected quasisplit of type ${ }^{2} A_{6}$. Then the hermitian form $h=\langle-1,-1,-1,-1,-1,-1,-1\rangle$ has trace form $q=-7 \ll-1 \gg$ which is not hyperbolic, so $h$ corresponds to a (unique) nontrivial class in $H^{1}(F, G)$. However,

$$
q-q^{d}=-7 \ll-1 \gg-\ll-1 \gg=-\ll-1,-1,-1,-1 \gg \in I^{4} F,
$$

so $R_{G}(h)$ is trivial.

\section{Folded root systems}

2.1. The Rost multiplier. A loop in an arbitrary algebraic group $G$ is a homomorphism $\mathbb{G}_{m} \rightarrow G$. Let $G_{*}$ be the set of loops in $G$. As in [KMRT98, p. 432], we set $Q(G)$ to be the abelian group of all integer-valued functions on $G_{*}$ such that

(1) for ${ }^{g} f$ the loop given by ${ }^{g} f(x)=g f(x) g^{-1}, q\left({ }^{g} f\right)=q(f)$ for all $g \in G$ and $f \in G_{*} ;$ and

(2) for any two loops $f$ and $h$ with commuting images, the function $\mathbb{Z} \times \mathbb{Z} \longrightarrow \mathbb{Z}$ given by $(k, m) \mapsto q\left(f^{k} h^{m}\right)$ is a quadratic form.

When $G$ is a simple group, $Q(G)$ is cyclic with a canonical generator which is positive definite [KMRT98, 31.27], hence is identified with $\mathbb{Z}$. Now suppose that we have two simple simply connected groups $H \subset G$. The inclusion gives a map $H_{*} \rightarrow G_{*}$, so we in turn have a map $\mathbb{Z}=Q(G) \rightarrow Q(H)=\mathbb{Z}$. Because the canonical generators are positive definite, this map must be multiplication by a positive integer $n$, which we define to be the Rost multiplier of the inclusion.

The naturality of the Rost invariant implies that we have a commutative diagram

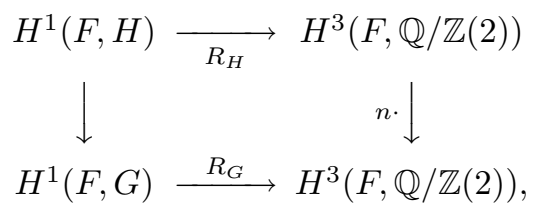

where $n$ is the Rost multiplier of the inclusion [KMRT98, 31.34]. This is the motivation for our study of this invariant. 
2.2. Luckily, it can be quite easy to compute such a "Rost multiplier". Suppose that $G$ and $H$ are split and contain split maximal tori $S$ and $T$ respectively such that the $T$ lies in $S$. Since $G$ and $H$ are simply connected, the character groups $X(T)$ and $X(S)$ are identified with the weight lattices, but the character groups are dual to the loop groups $S_{*}$ and $T_{*}$ [Bor91, 8.6] and the weight lattices are dual to the lattices generated by the coroots, which we denote by $\Lambda_{c, G}$ and $\Lambda_{c, H}$, respectively. (By a coroot, we mean the roots of the dual root system, which are denoted by $\check{\alpha}$ in [Bou68, VI.1] for $\alpha$ a root.) Putting these dualities together, we obtain identifications $S_{*}=\Lambda_{c, G}$ and $T_{*}=\Lambda_{c, H}$, so the inclusion $T \subset S$ induces a map $\Lambda_{c, H} \rightarrow \Lambda_{c, G}$. Now the dual root systems (whose roots are the coroots) are indeed root systems [Bou68, VI.1.1, Prop. 2] and so they each have a unique minimal Weyl-group invariant positive-definite integer-valued quadratic form [Bou68, VI.1.2, Prop. 7], say $q$ and $r$ (for the forms for $G$ and $H$ respectively). Hence $q$ induces such a form on $\Lambda_{c, H}$, which must be of the form $n r$ for some natural number $n$. This $n$ is the Rost multiplier of the inclusion.

Criterion (2) in the definition of $Q(G)$ implies that its canonical generator is identified with the positive-definite Weyl-group invariant quadratic form on the dual root system which takes the value 1 on short coroots. (Short roots correspond to long roots, where we adopt the convention that short = long in the event that all roots have the same length. In that case, the quadratic form is very easy to identify, in that its Gram matrix is simply the Cartan matrix of the root system with all entries divided by 2.) So one can simply compute the image of a short coroot from $H$ in the dual root system for $G$ to find the Rost multiplier of the inclusion.

Example 2.3 $\left(S L_{n} \rightarrow S L_{2 n}\right)$. The block diagonal embedding $S L_{n} \hookrightarrow S L_{2 n}$ via $x \mapsto\left(\begin{array}{c}{ }^{x} \\ x\end{array}\right)$ has Rost multiplier 2. The map given by $x \mapsto\left({ }^{x}{ }_{1}\right)$ has Rost multiplier 1 .

Example 2.4 (Folding). The split simply connected group of type $E_{6}$ can be realized as the group Inv $(J)$ of invertible linear maps of the split Albert algebra $J$ which preserve the cubic norm form. The algebra $J$ has a nondegenerate symmetric bilinear trace form $T$ given by setting $T(x, y)$ to be the trace of the product $x \cdot y$ [Jac68, p. 240, Thm. 5], and for $\varphi \in \operatorname{Inv}(J)(F)$ we define $\varphi^{\dagger} \in G L(J)(F)$ to be the unique map satisfying $T\left(\varphi(j), \varphi^{\dagger}\left(j^{\prime}\right)\right)=T\left(j, j^{\prime}\right)$ for all $j, j^{\prime} \in J$. This defines an outer automorphism of $E_{6}=\operatorname{Inv}(J)$ [Jac61, p. 76, Prop. 3] and the subgroup of elements fixed by this automorphism is the split group $F_{4}$ of $F$-algebra automorphisms of $J$.

We would like to compute the Rost multiplier of the inclusion $F_{4} \subset E_{6}$. Fix an $F$-split maximal torus $S$ in $G:=E_{6}$ which is preserved by the automorphism (such as the one denoted by " $S_{6}$ " in [Gar01b, pf. of 7.2]) and fix a set of simple roots $\Delta$ of $G$ with respect to $S$. We would like our outer automorphism to leave $\Delta$ invariant, although it probably does not do so. However, two things are apparent 
from the definition of the Rost multiplier: it is not changed by scalar extension nor by modifying the automorphism $\varphi \mapsto \varphi^{\dagger}$ by an inner automorphism of $E_{6}$. So we may assume that the base field is separably closed and so that the $F$-points of the Weyl group of $G$ with respect to $S$ (i.e., the $F$-points of $N_{G}(S) / S$ ) is the full Weyl group of the root system of $G$ with respect to $S$. Then we may modify our outer automorphism by an element of the Weyl group so that $F_{4}$ is described as the subgroup of $E_{6}$ fixed by the automorphism $f$ induced by the automorphism of $\Delta$ which is given by the unique nontrivial automorphism of the Dynkin diagram. That is, we set $H:=F_{4}=G^{f}$ (= the subgroup of $G$ of elements fixed by $f$ ), and $T:=\left(S^{f}\right)^{\circ}$ (= the identity component of $\left.T \cap G^{f}\right)$ is a maximal torus in $H$. The restrictions of elements of $\Delta$ to $T$ give a root system of $H$ with respect to $T$ [Sch69, p. 108] and the fibers of this restriction map are the orbits of $f$ in $\Delta$ [Sch69, 3.5].

Now $\Lambda_{c, G}$ is a free $\mathbb{Z}$-module with basis $\check{\Delta}=\{\check{\delta} \mid \delta \in \Delta\}$ which is permuted by $f$ and $\Lambda_{c, H}$ is the fixed sublattice. So $\Lambda_{c, H}$ has a basis consisting of one element for each orbit of $f$ in $\breve{\Delta}$, and this element is given by the sum of the elements in the orbit in $\check{\Delta}$. There is a coroot $\check{\delta} \in \check{\Delta}$ which is fixed by $f$, hence $\check{\delta}$ is a member of the $\mathbb{Z}$-basis for $\Lambda_{c, H}$. The form $q$ on $\Lambda_{c, G}$ restricts to a positive-definite Weyl-invariant form on $\Lambda_{c, H}$ such that $q(\check{\delta})=1$, consequently $q$ restricts to be the minimal such form $r$. By the discussion in 2.2 the Rost multiplier of the inclusion $F_{4} \subset E_{6}$ is 1 .

Remark 2.5. Presumably this same argument also works in the other instances where one obtains a root system by "folding up" another root system all of whose roots have the same length, i.e., $C_{\ell+1} \subset A_{2 \ell+1}, B_{n-1} \subset D_{n}$, and $G_{2} \subset D_{4}$. The other root system consisting of roots of the same length, $A_{2 \ell}$, folds up to give the smaller root system $B C_{\ell}$, see [Hec84, Table I].

\section{Small representations}

We say a representation $V$ of an algebraic group $G$ is small if $G$ has an open orbit in $\mathbb{P}(V)$. We are interested in small representations in the case where $G$ is simple, which have all been classified as a consequence of the (more general) classification of prehomogeneous vector spaces, see [Kim88] for a survey. These small representations also provide "standard relative sections" in the language of [Pop94, 1.7], and in that sense were classified in [Èla72, Table 1]. Our motivation for studying these representations comes from the following easy lemma, which was pointed out to me by Rost.

Lemma 3.1. Suppose that $G$ is an algebraic group over a field $F$ such that $G$ has a small representation $V$, and that $F$ is infinite or $G$ is connected. Let $H$ be a subgroup of $G$ consisting of the elements which stabilize some F-point in the open 
orbit in $\mathbb{P}(V)$. Then the natural map

$$
H^{1}(F, H) \rightarrow H^{1}(F, G)
$$

is surjective.

Proof. If the base field $F$ is finite, then by hypothesis $G$ is connected, and by Lang's Theorem $H^{1}(F, G)$ is trivial so the lemma holds. So we may assume that $F$ is infinite.

Fix a 1-cocycle $z \in Z^{1}(F, G)$. It defines a twisted version $\mathbb{P}(V)_{z}$ of $\mathbb{P}(V)$ which is the same as $\mathbb{P}(V)$ over the separable closure $F_{\text {sep }}$ of $F$ but has a different Galois action: For $w \in \mathbb{P}(V)_{z}\left(F_{\mathrm{sep}}\right)$ and $\sigma \in \operatorname{Gal}\left(F_{\mathrm{sep}} / F\right), \sigma$ acts by

$$
\sigma * w=z_{\sigma} \sigma w
$$

where juxtaposition denotes the usual action. The twisted version $U_{z}$ of $U$, defined analogously, is an open subset of $\mathbb{P}(V)_{z}$.

Since the representation gives a map $G \rightarrow G L(V), \mathbb{P}(V)_{z}$ is $F$-isomorphic to $\mathbb{P}(V)$. In particular, since $F$ is infinite, $\mathbb{P}(V)_{z}(F)$ is dense in $\mathbb{P}(V)_{z}\left(F_{\text {sep }}\right)$. Since $U_{z}\left(F_{\text {sep }}\right)$ is open in $\mathbb{P}(V)_{z}\left(F_{\text {sep }}\right)$, the two sets $U_{z}\left(F_{\text {sep }}\right)$ and $\mathbb{P}(V)_{z}(F)$ must meet nontrivially, i.e., $U_{z}$ has some $F$-point which we will denote by $x_{z}$.

Now let $x \in U(F)$ be the point with stabilizer subgroup $H$ and fix some $g \in G\left(F_{\mathrm{sep}}\right)$ such that $g x=x_{z}$. Then for all $\sigma \in \operatorname{Gal}\left(F_{\mathrm{sep}} / F\right)$, the element $g^{-1} z_{\sigma}(\sigma g)$ fixes $x$ and so lies in $H\left(F_{\text {sep }}\right)$. Thus $z$ is cohomologous to something in the image of $Z^{1}(F, H)$.

Example 3.2 $\left(O_{n-1} \subset O_{n}\right)$. Write $O_{n}$ for the orthogonal group of the dot product on $F^{n}$. Then the subgroup of $O_{n}$ which stabilizes $[v] \in \mathbb{P}\left(F^{n}\right)$ where $v$ has nonzero length is just $O_{n-1} \times \boldsymbol{\mu}_{2}$, where $O_{n-1}$ is the orthogonal group for the $(n-1)$ dimensional space of vectors in $F^{n}$ which are orthogonal to $v$. Iterating this process recovers the fact that all nondegenerate quadratic forms are diagonalizable, a.k.a. the Spectral Theorem.

Example 3.3 $\left(\operatorname{Spin}_{n}\right.$ [Igu70], [GV78], [Pop80]). For $\operatorname{Spin}_{n}$ the spin group for an $n$-dimensional maximally split quadratic form, the spin representation (if $n$ is odd) or the half-spin representation (if $n$ is even) is small for $n \leq 12$ and $n=14$. In the $n=14$ case, the stabilizer subgroup is isomorphic to $\left(G_{2} \times G_{2}\right) \rtimes \boldsymbol{\mu}_{8}$, and this leads to structural statements about 14 -dimensional forms in $I^{3} F$, see [Ros99].

Example 3.4 $\left(F_{4} \times \boldsymbol{\mu}_{3} \subset E_{6}\right)$. We write $E_{6}$ for the split group of type $E_{6}$ which can be realized as Inv $(J)$ as described in 2.4. By [Jac61, p. 71, Thm. 7], $E_{6}$ acts transitively on the subset of $J$ consisting of elements of norm 1, so certainly this is a small representation.

Take $H$ to be the subgroup of $E_{6}$ consisting of elements which fix the identity element $1_{J}$ of $J$ projectively. Since the norm form is cubic, $\boldsymbol{\mu}_{3}$ is contained in $H$ and is central (since it consists of scalar endomorphisms), and any element 
of $H$ differs by an element of $\boldsymbol{\mu}_{3}$ from something which fixes $1_{J}$ absolutely. This subgroup of elements fixing $1_{J}$ is well-known - it is the automorphism group $F_{4}$ of $J$ [Jac59, p. 186, Thm. 4], which is split of type $F_{4}$. So $H$ is isomorphic to $F_{4} \times \boldsymbol{\mu}_{3}$, and the resulting surjective map $H^{1}\left(F, F_{4} \times \boldsymbol{\mu}_{3}\right) \rightarrow H^{1}\left(F, E_{6}\right)$ is the statement that $H^{1}\left(F, E_{6}\right)$ classifies cubic forms of the form $\lambda N$ for $N$ the norm form on some Albert $F$-algebra and $\lambda \in F^{*}$, see [Spr62]. This can also be interpreted in terms of structurable algebras, see [Gar01b, 2.8(1)].

Example 3.5 $\left(E_{6} \rtimes \boldsymbol{\mu}_{4} \subset E_{7}\right)$. Write $E_{7}$ for the split simply connected group of type $E_{7}$ over $F$. It is the group of vector space automorphisms of $V=\left(\begin{array}{cc}F \\ J\end{array}\right)$ which preserve a quartic form $q$ as given in [Bro69, p. 87]. Then $E_{7}$ acts transitively on the open subset of $\mathbb{P}(V)$ consisting of points $[v]$ such that $q(v) \neq 0$ by [Fer72, 7.7].

We set $H$ to be the subgroup of $E_{7}$ which stabilizes the vector $v=\left(\begin{array}{ll}1 & 0 \\ 0 & 1\end{array}\right)$ projectively. This vector has $q(v) \neq 0$, and so by [Fer72,3.7] there are two uniquely determined (up to scalar multiples) "strictly regular" elements $e_{1}$ and $e_{2}$ such that $v$ lies in their span. These are $e_{1}=\left(\begin{array}{ll}1 & 0 \\ 0 & 0\end{array}\right)$ and $e_{2}=\left(\begin{array}{ll}0 & 0 \\ 0 & 1\end{array}\right)$. Since $E_{7}$ preserves the property of being strictly regular, every element of $H$ must projectively stabilize $e_{1}$ and $e_{2}$ as well, and perhaps interchange them.

Now, the map $\omega$ defined by

$$
\omega\left(\begin{array}{cc}
\alpha & j \\
j^{\prime} & \beta
\end{array}\right)=\left(\begin{array}{cc}
i \beta & i j^{\prime} \\
i j & i \alpha
\end{array}\right)
$$

lies in $H$, where $i$ is some fixed square root of -1 in the separable closure of $F$. We would like to describe an arbitrary $h \in H$, which after modification by $\omega$ we may assume projectively stabilizes each of $e_{1}$ and $e_{2}$. Then by [Bro69, p. 96, Lem. 12], $h$ must be of the form

$$
h\left(\begin{array}{cc}
\alpha & j \\
j^{\prime} & \beta
\end{array}\right)=\left(\begin{array}{ll}
\mu^{-1} \alpha & \varphi(j) \\
\varphi^{\dagger}\left(j^{\prime}\right) & \mu \beta
\end{array}\right)
$$

where $\varphi$ is a similarity of the norm form on $J$ with multiplier $\mu$ and $\varphi^{\dagger}$ is as defined in 2.4. Since $h$ also stabilizes $v$, we must have that $\mu= \pm 1$. In particular, after modifying $h$ by $\omega^{2}=-1$, we may assume that $h$ has the form

$$
h\left(\begin{array}{cc}
\alpha & j \\
j^{\prime} & \beta
\end{array}\right)=\left(\begin{array}{cc}
\alpha & \varphi(j) \\
\varphi^{\dagger}\left(j^{\prime}\right) & \beta
\end{array}\right)
$$

where $\varphi$ preserves the cubic norm on $J$ and so lies in $E_{6}$. We have shown that $H$ is isomorphic to $E_{6} \rtimes \boldsymbol{\mu}_{4}$.

The surjection on Galois cohomology coming from this example will be more useful if we can replace $E_{6} \rtimes \boldsymbol{\mu}_{4}$ with a simple group. For $K$ a quadratic étale $F$-algebra, we write $E_{6}^{K}$ for the simply connected quasi-split group of type $E_{6}$ over $F$ which is split by an extension $L$ of $F$ if and only if $L \otimes_{F} K \cong L \times L$.

Proposition 3.6 (Cf. [Gar01b, 4.14]). For each $\alpha \in H^{1}\left(F, E_{7}\right)$ there is some quadratic étale $F$-algebra $K$ such that $E_{6}^{K}$ embeds in $E_{7}$ with Rost multiplier 1 and $\alpha$ is in the image of the induced map $H^{1}\left(F, E_{6}^{K}\right) \rightarrow H^{1}\left(F, E_{7}\right)$. 
Proof. Fix some $a \in Z^{1}\left(F, E_{6} \rtimes \boldsymbol{\mu}_{4}\right)$ representing $\alpha$. The natural projection $E_{6} \rtimes$ $\boldsymbol{\mu}_{4} \rightarrow \boldsymbol{\mu}_{4}$ has an obvious section given by sending $i \mapsto \omega$; set $b$ to be the image of $a$ given by the map induced by the composition $E_{6} \rtimes \boldsymbol{\mu}_{4} \rightarrow \boldsymbol{\mu}_{4} \rightarrow E_{6} \rtimes \boldsymbol{\mu}_{4}$. Twist $E_{6} \rtimes \boldsymbol{\mu}_{4}$ by $b$ to obtain a new group $\left(E_{6} \rtimes \boldsymbol{\mu}_{4}\right)_{b}$, with a twisted Galois action $*$ so that

$$
\sigma * g=b_{\sigma}\left({ }^{\sigma} g\right) b_{\sigma}^{-1}
$$

where ${ }^{\sigma} g$ denotes the usual action. There is an isomorphism

$$
H^{1}\left(F,\left(E_{6} \rtimes \boldsymbol{\mu}_{4}\right)_{b}\right) \underset{\tau_{b}}{\stackrel{\sim}{\longrightarrow}} H^{1}\left(F, E_{6} \rtimes \boldsymbol{\mu}_{4}\right)
$$

where $\tau_{b}^{-1}(\alpha)$ is the class of a 1-cocycle given by $\sigma \mapsto a_{\sigma} b_{\sigma}^{-1}$ with values in the identity component of the twisted group $\left(E_{6} \rtimes \boldsymbol{\mu}_{4}\right)_{b}$. This identity component is just $E_{6}$ twisted by $b$, and we would like to show that it is isomorphic to $E_{6}^{K}$ for some quadratic étale $F$-algebra $K$. If $\sigma$ in $\operatorname{Gal}\left(F_{\text {sep }} / F\right)$ has $b_{\sigma}= \pm 1$, then $\sigma$ acts in the usual manner upon the twisted $E_{6}$. On the other hand, if $b_{\sigma}= \pm \omega$, then the twisted action is given by

$$
(\sigma * h)\left(\begin{array}{cc}
\alpha & j \\
j^{\prime} & \beta
\end{array}\right)=( \pm \omega) \sigma h \sigma^{-1}( \pm \omega)^{-1}\left(\begin{array}{ll}
\alpha & j \\
j^{\prime} & \beta
\end{array}\right)=\left(\begin{array}{cc}
\alpha & \sigma \varphi^{\dagger} \sigma^{-1}\left(j^{\prime}\right) \\
\sigma \varphi \sigma^{-1}(j) & \beta
\end{array}\right) .
$$

This is precisely the description of the Galois action on $E_{6}^{K}$ given in [Gar01b, 2.4] for $K$ determined by the image of $b$ under the composition $H^{1}\left(F, E_{6} \rtimes \boldsymbol{\mu}_{4}\right) \rightarrow$ $H^{1}\left(F, \boldsymbol{\mu}_{4}\right) \rightarrow H^{1}\left(F, \boldsymbol{\mu}_{2}\right)=F^{*} / F^{* 2}$, so $\left(E_{6}\right)_{b}$ is isomorphic to $E_{6}^{K}$. To see that $E_{6}^{K}$ embeds in $E_{7}$, we observe that the 1-cocycle $b$ is trivial in $H^{1}\left(F, E_{7}\right)$ by [Gar01b, $4.10,5.10]$, so we have a map

$$
E_{6}^{K} \subset\left(E_{6} \rtimes \boldsymbol{\mu}_{4}\right)_{b} \hookrightarrow\left(E_{7}\right)_{b} \underset{f}{\stackrel{\sim}{\longrightarrow}} E_{7}
$$

where (by a simple computation having nothing to do with $\left.E_{7}\right) H^{1}(f)=\tau_{b}$. This proves the proposition aside from the claim about the Rost multiplier.

But that claim is easy in the split case (where $K=F \times F$ ), since the embedding of $E_{6}$ in $E_{7}$ comes from the obvious embedding of root systems. Since the Rost multiplier is invariant under scalar extension, the embeddings of quasi-split groups of type $E_{6}^{K}$ in $E_{7}$ given above all have Rost multiplier 1 as well.

\section{4. ${ }^{1} D_{4} \subset{ }^{2} E_{6}$}

For the remainder of the paper we will study the quasi-split group $E_{6}^{K}$ of type ${ }^{2} E_{6}$ defined in 3.5. In this section we introduce a particular subgroup $G$ of $E_{6}^{K}$ which is reductive of semisimple type ${ }^{1} D_{4}$. Defining $G$ will necessitate digging more deeply in to the structure of Cayley and Albert algebras.

Definition 4.1. Fix $\mathfrak{C}$ to be the split Cayley algebra endowed with hyperbolic norm form $\mathfrak{n}$ and canonical involution - ${ }^{-}$(For more information about Cayley 
algebras, see [KMRT98, §33.C] or [Sch66, Ch. III, §4].) If $t \in G L(\mathfrak{C})(F)$ satisfies $\mathfrak{n}(t(c))=m \mathfrak{n}(c)$ for some $m \in F^{*}$ and all $c \in \mathfrak{C}$, we say that $m$ is a similarity of $\mathfrak{n}$ with multiplier $\mu(t):=m$. (Note that if $\sigma_{\mathfrak{n}}$ is the involution on $\operatorname{End}_{F}(\mathfrak{C}$ ) which is adjoint for $\mathfrak{n}$ so that $\mathfrak{n}\left(t c, c^{\prime}\right)=\mathfrak{n}\left(c, \sigma_{\mathfrak{n}}(t) c^{\prime}\right)$ for all $c, c^{\prime} \in \mathfrak{C}$, then $\mu(t)=\sigma_{\mathfrak{n}}(t) t$. $)$ Set $G O^{\circ}(\mathfrak{C}, \mathfrak{n})$ to be the algebraic group with $F$-points

$$
G O^{\circ}(\mathfrak{C}, \mathfrak{n})(F):=\left\{\begin{array}{l|l}
t \in G L(\mathfrak{C})(F) & \begin{array}{l}
t \text { is a similarity of } \mathfrak{n} \text { with multiplier } \\
\mu(t) \text { such that } \operatorname{det}(t)=\mu(t)^{4}
\end{array}
\end{array}\right\}
$$

We can also define a new, seemingly uglier multiplication $\star$ on $\mathfrak{C}$ by setting $x \star$ $y:=\bar{x} \bar{y}$ as in [KMRT98, $\S 34 . \mathrm{A}]$. A related triple is a triple $\left(t_{0}, t_{1}, t_{2}\right)$ in $G O^{\circ}(\mathfrak{C}, \mathfrak{n})^{\times 3}$ such that

$$
\mu\left(t_{i}\right)^{-1} t_{i}(x \star y)=t_{i+2}(x) \star t_{i+1}(y)
$$

for all $x, y \in \mathfrak{C}$ and $i=0,1,2$ with subscripts taken modulo 3 . Write $\operatorname{Rel}(\mathfrak{C}, \mathfrak{n})$ for the algebraic subgroup of $G O^{\circ}(\mathfrak{C}, \mathfrak{n})^{\times 3}$ consisting of related triples and $\operatorname{Spin}(\mathfrak{n})$ for the subgroup of $\operatorname{Rel}(\mathfrak{C}, \mathfrak{n})$ consisting of triples with multiplier one (i.e., those triples such that $\mu\left(t_{i}\right)=1$ for all $\left.i\right)$.

4.2. The vector space underlying the split Albert $F$-algebra $J$ is the subspace of $M_{3}(\mathfrak{C})$ consisting of elements fixed by the conjugate transpose $*$ which applies - to each entry and takes the transpose. It is the algebra denoted by $\mathfrak{H}\left(\mathfrak{C}_{3}\right)$ in the notation of [Jac68, §I.5] and has multiplication $a \cdot b:=(a b+b a) / 2$, where juxtaposition denotes the usual multiplication on $M_{3}(\mathfrak{C})$. When writing down explicit elements of $J$, we will use a "." to indicate entries whose values are forced by this symmetry condition. The reductive group $\operatorname{Rel}(\mathfrak{C}, \mathfrak{n})$ embeds in the group Inv $(J)$ of norm isometries of $J$ via the map $\underline{t} \mapsto g_{\underline{t}}$ given by

$$
g_{\underline{t}}\left(\begin{array}{ccc}
\varepsilon_{0} & c_{2} & \cdot \\
\cdot & \varepsilon_{1} & c_{0} \\
c_{1} & \cdot & \varepsilon_{2}
\end{array}\right)=\left(\begin{array}{ccc}
\mu\left(t_{0}\right)^{-1} \varepsilon_{0} & t_{2}\left(c_{2}\right) & \cdot \\
\cdot & \mu\left(t_{1}\right)^{-1} \varepsilon_{1} & t_{0}\left(c_{0}\right) \\
t_{1}\left(c_{1}\right) & \cdot & \mu\left(t_{2}\right)^{-1} \varepsilon_{2}
\end{array}\right) .
$$

Let $e_{i}$ denote the element of $J$ whose only nonzero entry is a 1 in the $(i+1, i+1)$ position. Any element of $\operatorname{Inv}(J)(K)$ which fixes $e_{1}, e_{2}$, and $e_{3}$ is of the form $g_{\underline{t}}$ for some $\underline{t} \in \operatorname{Spin}(\mathfrak{n})$ by [Sod66, p. 155, Thm. 1]. This implies that every element of $\operatorname{Inv}(J)(F)$ which leaves each of subspaces $F e_{i}$ invariant is in the image of $\operatorname{Rel}(\mathfrak{C}, \mathfrak{n})$.

4.4. Definition of $G$. Since Rel $(\mathfrak{C}, \mathfrak{n})$ embeds in $\operatorname{Inv}(J)$ over $F$, it embeds in $E_{6}^{K}$ over $K$. However, we can identify $E_{6}^{K}$ with $\operatorname{Inv}(J)$ with a different $\iota$-action where ${ }^{\iota} f:=\iota f^{\dagger} \iota$, where $\iota$ is the nontrivial $F$-automorphism of $K$ and juxtaposition denotes the usual action; we fix this identification for the rest of the paper. The $\operatorname{map} \operatorname{Rel}(\mathfrak{C}, \mathfrak{n}) \rightarrow E_{6}^{K}$ is not defined over $F$ : For $\underline{t}=\left(t_{0}, t_{1}, t_{2}\right) \in \operatorname{Rel}(\mathfrak{C}, \mathfrak{n})(K)$ and $g_{\underline{t}} \in E_{6}^{K}$, we have ${ }^{\iota} g_{\underline{t}}=g_{\iota \sigma_{\mathfrak{n}}(\underline{t})^{-1} \iota}$ which is typically not the same as $g_{\iota \underline{t} \iota}$ where $\sigma_{\mathfrak{n}}(\underline{t})$ means to apply $\sigma_{\mathfrak{n}}$ to each component of $\underline{t}$. Define $G$ to be the algebraic group over $F$ which is the same as $\operatorname{Rel}(\mathfrak{C}, \mathfrak{n})$ over $K$ but with a different $\iota$-action: 
for $\underline{t} \in G(K)$, set ${ }^{\iota} \underline{t}:=\iota \sigma_{\mathfrak{n}}(\underline{t})^{-1} \iota$. Then $G$ injects into $E_{6}^{K}$ over $F$ via the map $g$ from (4.3).

This group $G$ is reductive with absolute rank 6 and semisimple part $\operatorname{Spin}(\mathfrak{n})$ of type ${ }^{1} D_{4}$.

4.5. The center $P$ of $G$. Set $N_{1}$ to be the algebraic group with $F$-points the elements of $K^{*}$ with norm 1 in $F$. This group is the same as $\mathbb{G}_{m}$ over $K$, but has a different $\iota$-action given by ${ }^{\iota} \lambda=\iota(\lambda)^{-1}$. It is sometimes denoted by $R_{K / F}^{(1)}\left(\mathbb{G}_{m, K}\right)$.

The center of $\operatorname{Rel}(\mathfrak{C}, \mathfrak{n})$ is the subgroup of $\mathbb{G}_{m}^{\times 3}$ consisting of triples whose product is one. But we are concerned with $G$, which has a different $\iota$-action; its center $P$ is isomorphic to the subgroup of $N_{1}^{\times 3}$ consisting of triples whose product is 1 . This rank 2 torus is $F$-anisotropic and $K$-split.

The importance of $G$ is given by the following lemma, excavated from a paper by Ferrar:

Ferrar's Lemma 4.6. [Fer69, p. 65, Lem. 3] The natural map $H^{1}(K / F, G) \rightarrow$ $H^{1}\left(K / F, E_{6}^{K}\right)$ is surjective.

Comments. Ferrar proved this by explicit computations in the Jordan algebra. However, this can also be seen with more algebraic group-theoretic methods, as was pointed out to me by Gille. We must assume that our base field has characteristic 0 , which as was observed in the introduction does not harm our main results in any way.

The group $\operatorname{Spin}_{8}$ is split simply connected of type $D_{4}$ and so contains a subgroup which is isogenous to $S L_{2}^{\times 4}$. Each copy of the group $S L_{2}$ contains a rank 1 torus which is anisotropic over $F$ and split over $K$, and we set $T_{4}$ to be the image in $\operatorname{Spin}_{8}$ of these four tori. Let $T$ be the subtorus of $G$ generated by $T_{4}$ and the center $P$. It is a rank $6 F$-anisotropic torus which is split over $K$. Let $B$ be a Borel subgroup of $E_{6}^{K}$ defined over $K$ and containing $T$. Then ${ }^{\iota} B \cap B=T$, so by [PR94, p. 369, Lem. 6.28] the natural map $H^{1}(K / F, T) \rightarrow H^{1}\left(K / F, E_{6}^{K}\right)$ is a surjection.

Now imagine how the argument for proving the main theorem in the ${ }^{2} E_{6}$ case must proceed: We apply some simple argumentation and Ferrar's Lemma to show that any class in $H^{1}\left(F, E_{6}^{K}\right)$ with trivial Rost invariant must come from $H^{1}(K / F, G)$. Then we apply some facts about Rost invariants on this smaller group to obtain the theorem. However, $G$ is reductive, so we want to put our class with trivial Rost invariant into a simple subgroup if we hope to apply our results from Section 1. This requires further study of the center of $G$.

4.7. The group $H^{1}(K / F, P)$. There is a short exact sequence over $K$

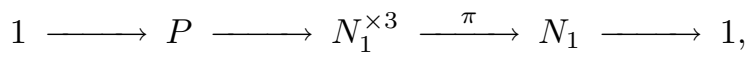


where $\pi$ is the product map, which induces an exact sequence

$$
H^{1}(K / F, P) \longrightarrow H^{1}\left(K / F, N_{1}^{\times 3}\right) \stackrel{H^{1}(\pi)}{\longrightarrow} H^{1}\left(K / F, N_{1}\right) .
$$

The first map is an injection because the product map $\pi$ is a surjection on $F$ points. Any 1-cocycle in $Z^{1}\left(K / F, N_{1}\right)$ is determined by its value at $\iota$, and the condition that it is a 1-cocycle forces that this value lies in $F^{*}$. The obvious check shows that two such are cohomologous if and only if they differ by a norm from $K^{*}$. So $H^{1}(K / F, P)$ is isomorphic to the subgroup of $\left(F^{*} / N_{K / F}\left(K^{*}\right)\right)^{\times 3}$ consisting of elements with product in $N_{K / F}\left(K^{*}\right)$.

4.8. The map $H^{1}(K / F, G) \rightarrow H^{1}(K / F, P)$. There is a short exact sequence

$$
1 \longrightarrow \operatorname{Spin}(\mathfrak{n}) \longrightarrow P \longrightarrow 1
$$

where the map $G \rightarrow P$ is given by sending each $t_{i}$ to its multiplier $\mu\left(t_{i}\right)=\sigma_{\mathfrak{n}}\left(t_{i}\right) t_{i} \in$ $N_{1}$. This sequence is even exact over $K$ (instead of just over a separable closure of $F$ ) because the map $G \rightarrow P$ is surjective over $K$ by [KMRT98, 35.4]. A 1cocycle $\gamma \in Z^{1}(K / F, G)$ is determined by its value $\gamma_{\iota}$ at $\iota$, and the image of $\gamma$ in $H^{1}(K / F, P)$ is the multiplier of $\gamma_{\iota}$.

A natural question is the following: Any 1-cocycle in $Z^{1}\left(K / F, E_{6}^{K}\right)$ comes from $H^{1}(K / F, G)$ by Ferrar's Lemma and so has an image in $H^{1}(K / F, P)$. Is that image an invariant of the original class in $H^{1}\left(K / F, E_{6}^{K}\right)$ ? The answer is no, as is shown in the following lemma. (Explicit situations where the hypotheses are satisfied nontrivially will be given in 6.6 and 7.10 .)

Moving Lemma 4.9. Let $\eta$ be a 1-cocycle in $Z^{1}(K / F, G)$ whose image in $Z^{1}(K / F, P)$ takes the value $\underline{a}$ at $\iota$. Suppose that there is some $j \in e_{0} \times J_{K}$ such that

$$
j^{\#}=0 \quad \text { and } \quad T\left(j, \eta_{\iota} \iota j\right)=r \in F^{*} .
$$

Then $\eta$ is cohomologous in $H^{1}\left(K / F, E_{6}^{K}\right)$ to a 1-cocycle coming from $Z^{1}(K / F, G)$ whose image in $Z^{1}(K / F, P)$ takes the value $\left(r^{-1}, a_{0}, a_{0}^{-1} r\right)$ at $\iota$.

The hypotheses in the lemma make use of the Freudenthal cross product $\times$ : $J \times J \rightarrow J$, which is a commutative bilinear map defined by the relation $6 N(j)=$ $T(j, j \times j)$ for all $j \in J$. The map $\#: J \rightarrow J$ is defined by $2 j \#:=j \times j$.

The proof is an adaptation of an argument in [Fer80, p. 277].

Proof. First observe that the three elements $j, e_{0}$, and $e_{0} \times j^{\prime}$ for $j^{\prime}:=\eta_{\iota} \iota j$ all have "rank one", i.e., are sent to zero by the map $x \mapsto x^{\#}$.

For $x$ and $y$ in $J$, we have the identity [McC69, (19)]:

$$
x \times\left(x^{\#} \times y\right)=N(x) y+T(x, y) x^{\#} .
$$

Setting $x=w+z$, we have $x^{\#}=w^{\#}+w \times z+z^{\#}$. The term of $N(x)=N(w+z)$ which has degree 1 in $w$ and degree 2 in $z$ is $T\left(w, z^{\#}\right) y$. Substituting $x=w+z$ 
into (4.10) and taking the terms on both sides with this degree, we obtain the identity

$$
\begin{aligned}
& w \times\left(z^{\#} \times y\right)+z \times(y \times(z \times w))= \\
& T\left(w, z^{\#}\right) y+T(z, y)(w \times z)+T(w, y) z^{\#} .
\end{aligned}
$$

Since the vector space $e_{0} \times J$ is preserved by $\iota$ and $G(K)$, we have $j^{\prime}=e_{0} \times w$ for some $w$. Applying (4.11), we obtain

$$
e_{0} \times\left(e_{0} \times j^{\prime}\right)=T\left(e_{0}, e_{0}\right)\left(e_{0} \times y\right)=j^{\prime} .
$$

For $N$ trilinearized so that $N(x, x, x)=N(x)$, we have

$$
6 N\left(e_{0}, j, e_{0} \times j^{\prime}\right)=T\left(e_{0} \times j, e_{0} \times j^{\prime}\right)=T\left(j, e_{0} \times\left(e_{0} \times j^{\prime}\right)\right)=T\left(j, j^{\prime}\right)=r \neq 0 .
$$

(The triple $e_{0}, j, e_{0} \times j^{\prime}$ is said to be "in general position".) By [SV68, 3.11], this implies that there exist some $f \in \operatorname{Inv}(J)(K)$ and $\rho_{i} \in K^{*}$ such that

$$
f(j)=\rho_{0} e_{0}, \quad f\left(e_{0}\right)=\rho_{1} e_{1}, \text { and } f\left(e_{0} \times j^{\prime}\right)=\rho_{2} e_{2} .
$$

Since $\mathfrak{n}$ is hyperbolic, there is some $g=g_{\underline{t}} \in \operatorname{Inv}(J)(K)$ such that $g\left(e_{0}\right)=\rho_{0}^{-1} e_{0}$, $g\left(e_{1}\right)=\rho_{1}^{-1} e_{1}$, and $g\left(e_{2}\right)=\rho_{0} \rho_{1} e_{2}$. By replacing $f$ with $g f$, we may assume that $\rho_{0}=\rho_{1}=1$. Moreover, $f$ preserves $N$, and so $\rho_{2}=6 N\left(e_{0}, e_{1}, \rho_{2} e_{2}\right)=r$.

Set $\eta^{\prime} \in Z^{1}\left(K / F, E_{6}^{K}\right)$ to be the cocycle cohomologous to $\eta$ given by $\eta_{\iota}^{\prime}=$ $f^{\dagger} \eta_{\iota}{ }^{\iota}\left(f^{\dagger}\right)^{-1}$. It is standard that the maps $\dagger$ and inverse commute on $\operatorname{Inv}(J)$, hence ${ }^{\iota}\left(f^{\dagger}\right)^{-1}={ }^{\iota}\left(f^{-1}\right)^{\dagger}=\iota f^{-1} \iota$, where the action of $\iota$ on $E_{6}^{K}$ is as in 4.4. Thus we have

$$
\eta_{\iota}^{\prime}=f^{\dagger} \eta_{\iota} \iota f^{-1} \iota
$$

Keeping in mind the facts that $e_{i} \times e_{i+1}=e_{i+2} ; f^{\dagger}(u \times v)=f(u) \times f(v)$ for all $u, v \in J_{K}$; equation (4.12); and $j \times\left(e_{0} \times j^{\prime}\right)=r e_{0}$ (as can be verified by examining the explicit formula for $\times$ given in [Jac68, p. 358, (4)], although the reader should be cautioned that our definition of $\times$ - which agrees with the one in [KMRT98] and [McC69] — differs from Jacobson's by a factor of 2), one can now easily calculate that $f^{\dagger}\left(e_{0}\right)=e_{1}$ and $f^{\dagger}\left(j^{\prime}\right)=r e_{0}$. It follows that

$$
\eta_{\iota}^{\prime}\left(e_{0}\right)=r e_{0}, \quad \text { and } \quad \eta_{\iota}^{\prime}\left(e_{1}\right)=a_{0}^{-1} e_{1} .
$$

Since $\eta$ is a 1-cocycle, we have $\eta_{\iota} \iota(u \times v)=\left(\iota \eta_{\iota}^{-1} u\right) \times\left(\iota \eta_{\iota}^{-1} v\right)$. Thus $\eta_{\iota} \iota\left(e_{0} \times j^{\prime}\right)=$ $a_{0} e_{0} \times j$, and we have

$$
\eta_{\iota}^{\prime}\left(e_{2}\right)=\left(a_{0} / r\right) e_{2}
$$

Since $\eta_{\iota}^{\prime}$ preserves the linear subspaces $K e_{i}$ for all $i$, it belongs to $G(K)$, and we are done.

\section{5. ${ }^{2} D_{5} \subset{ }^{2} E_{6}$}

For the purpose of making computations, we will need to make use of another subgroup of $E_{6}^{K}$, which we define to be the subgroup consisting of elements $h$ such 
that $h$ and $h^{\dagger}$ both fix the element $e_{0} \in J$. Since the map $h \rightarrow h^{\dagger}$ is a group homomorphism on $\operatorname{Inv}(J)$, it is clear that $H$ is indeed a subgroup of $E_{6}^{K}$ over $K$, and it is preserved by the $\iota$-action so it is even defined over $F$. Our first task is to describe it explicitly.

5.1. Fix a particular basis $u_{1}, u_{2}, \ldots, u_{8}$ for the split Cayley algebra $\mathfrak{C}$ as given in [Gar98, p. 388]. One important thing for us to know about this basis is that when we bilinearize the norm form $\mathfrak{n}$ so that $\mathfrak{n}(x, x)=2 \mathfrak{n}(x)$, we have

$$
\mathfrak{n}\left(u_{i}, u_{j}\right)= \begin{cases}1 & \text { if } i+j=9 \\ 0 & \text { otherwise }\end{cases}
$$

so that the Gram matrix of the symmetric bilinear form with respect to this basis is a matrix we will denote by $S_{8}$. It is the $8 \times 8$ matrix which has zeroes everywhere except for a line of ones connecting the $(1,8)$ and the $(8,1)$ entries. Also, the canonical involution ${ }^{-}$is given by

$$
\overline{u_{i}}= \begin{cases}-u_{i} & \text { if } i \neq 4,5 \\ u_{5} & \text { if } i=4 \\ u_{4} & \text { if } i=5\end{cases}
$$

5.2. Over $K, H$ is isomorphic to $\operatorname{Spin}_{10} \rtimes \boldsymbol{\mu}_{2}$. Let $A$ denote the 10 -dimensional subspace $e_{0} \times J$ of $J$, which is $A=\left(\begin{array}{ccc}0 & 0 & \dot{C} \\ 0 & F & c \\ 0 & . & F\end{array}\right)$. For $f$ in $\operatorname{Inv}(J)(K)$, we have $f\left(e_{0} \times j\right)=f^{\dagger}\left(e_{0}\right) \times f^{\dagger}(j)$, so for $f \in H$ we have $f(A)=A$. The multiplication on $J$ restricts to give $A$ the structure of a central simple Jordan algebra as well, albeit with a different unit element. It has norm form $N_{A}$ given by

$$
N_{A}\left(\begin{array}{ccc}
0 & 0 & \dot{c} \\
\dot{0} & \alpha & c \\
0 & \cdot & \beta
\end{array}\right)=\alpha \beta-\mathfrak{n}(c) \text {. }
$$

Extend scalars to $K(t)$ and fix $f$ in $H(K)$. Then $N\left(t e_{0}+j\right)=N\left(f\left(t e_{0}+j\right)\right)=$ $N\left(t e_{0}+f(j)\right)$. The coefficient of $t$ in this expression is $T\left(e_{0}, j^{\#}\right)=T\left(e_{0}, f(j)^{\#}\right)$. For $j$ actually lying in $A, T\left(e_{0}, j^{\#}\right)=N_{A}(j)$, so $f$ restricts to preserve the norm on $A$. Write $O(A)$ for the algebraic subgroup of $G L(A)$ consisting of maps which preserve the norm $N_{A}$ (i.e., the orthogonal group of the 10-dimensional quadratic form $N_{A}$ ). We have proven that restriction provides a map $H \rightarrow O(A)$ which is defined over $K$.

The map $H \rightarrow O(A)$ has kernel of order 2: Anything in $H$ which maps to the identity in $O(A)$ fixes all of the idempotents $e_{i}$, and so is of the form $g_{t}$ for some $\underline{t} \in \operatorname{Spin}(\mathfrak{n})$. However, $t_{0}$ must also be the identity, so $\underline{t}=(1,1,1)$ or $(1,-1,-1)$ by $[$ Gar98, 1.5(2)].

We would like to show that the map $H \rightarrow O(A)$ is surjective. Note that $O(A)$ is generated by 
- the special orthogonal group $S O(B)$ for $B$ the codimension 1 subspace of $A$ spanned by $\mathfrak{C}$ and $e_{1}-e_{2}$ endowed with the quadratic form given by restricting $N_{A}$

- anything in $O(A)$ with determinant -1 ; and

- anything in $O(A)$ which does not leave $B$ invariant.

Since for $f \in \operatorname{Aut}(J), f^{\dagger}=f$, the subgroup $\operatorname{Aut}\left(J / e_{0}\right)$ of elements of $\operatorname{Aut}(J)$ which fix $e_{0}$ is a subgroup of $H$. As described in [Jac68, p. 376, Thm. 4], Aut $\left(J / e_{0}\right) \cong$ $\operatorname{Spin}(B)$ and the restriction to $B$ gives the surjection onto $S O(B)$. The map

$$
\left(\begin{array}{ccc}
\varepsilon_{0} & c_{2} & \cdot \\
\dot{c_{1}} & \varepsilon_{1} & c_{0} \\
c_{1} & \cdot & \varepsilon_{2}
\end{array}\right) \mapsto\left(\begin{array}{ccc}
\varepsilon_{0} & c_{1} & \cdot \\
\dot{c_{2}} & \varepsilon_{2} & \dot{c}_{0} \\
c_{2} & \cdot & \varepsilon_{1}
\end{array}\right)
$$

lies in $H(K)$ and restricts to have determinant -1 on $A$. Finally, we consider Freudenthal's maps from [Jac61, p. 74]. For $E_{i j} \in M_{3}(\mathfrak{C})$ the matrix whose only nonzero entry is a 1 in the $(i, j)$-position, $1_{3}$ the $3 \times 3$ identity matrix, $x \in \mathfrak{C}$, and $a \in J$, he defines a map $\psi_{i j}(x) \in \operatorname{Inv}(J)$ given by

$$
\psi_{i j}(x)(a)=\left(1_{3}+x E_{i j}\right) a\left(1_{3}+x E_{i j}\right)^{*},
$$

where juxtaposition denotes the usual multiplication in $M_{3}(\mathfrak{C})$, not the Jordan multiplication. So $\psi_{i j}(x) \in H(K)$ if $i, j \neq 1$. In particular, $\left.\psi_{32}\left(u_{5}\right)\right|_{A}$ is given by

$$
\left.\psi_{32}\left(u_{5}\right)\right|_{A}\left(\begin{array}{cc}
\varepsilon_{1} & c_{0} \\
\cdot & \varepsilon_{2}
\end{array}\right)=\left(\begin{array}{cc}
\varepsilon_{1} & c_{0}+\varepsilon_{1} u_{4} \\
\cdot & \varepsilon_{2}+\mathfrak{n}\left(c_{0}, u_{4}\right)
\end{array}\right),
$$

which does not leave $B$ invariant.

Finally, we observe that $H^{\circ}$ is isomorphic to $\operatorname{Spin}(A)$. The inverse image, call it $H^{\prime}$, of $S O(A)$ maps onto $S O(A)$ with a kernel which is central and of order 2. Consequently, $H^{\prime}$ is simple and hence must be isomorphic to $\operatorname{Spin}(A)$. Since $H^{\prime}$ is connected and $\left[H: H^{\prime}\right]=2, H^{\circ}=H^{\prime}$.

5.3. Over $F, H$ is isomorphic to $\operatorname{Spin}(4 \mathcal{H} \perp\langle-1, k\rangle) \rtimes \boldsymbol{\mu}_{2}$. To compute the isomorphism class of $H$ over $F$, we observe that the map $h \mapsto h^{\dagger}$ restricts to the identity on the kernel of the $K$-map $H \rightarrow O(A)$, so the $\iota$-action on $H$ induces one on $O(A)$, which we will calculate explicitly.

Fix the basis $\left(u_{1}, u_{2}, u_{3}, u_{4}, e_{1}, e_{2}, u_{5}, \ldots, u_{8}\right)$ for $A$ so that the Gram matrix for the symmetric bilinear form associated with $N_{A}$ becomes

$$
\left({ }_{-S_{4}} S^{-S_{4}}\right)
$$

for $S_{2}$ and $S_{4}$ defined analogously to how $S_{8}$ was in 5.1. Then $S O(A)$ is generated by

- a torus $T$ consisting of diagonal matrices with diagonal entries $\left(d_{1}, d_{2}, \ldots\right.$, $\left.d_{5}, d_{5}^{-1}, d_{4}^{-1}, \ldots, d_{1}^{-1}\right)$

- root groups $U_{i j}: \mathbb{G}_{a} \rightarrow S O(A)$ given by

$$
U_{i j}(r)=1_{10}+r E_{i j}-r E_{j^{*} i^{*}}
$$

for $1_{10}$ the $10 \times 10$ identity matrix, $i^{*}:=11-i$, and $(i, j)=(i, i+1)$ for $i=1,2,3$, and their transposes; and 
- root groups $V_{i j}: \mathbb{G}_{a} \rightarrow S O(A)$ given by

$$
V_{i j}(r)=1_{10}+r\left(E_{i j}+E_{j^{*} i^{*}}\right)
$$

for $(i, j)=(4,5)$ and $(4,6)$, and their transposes. (Note that $V_{45}(r)=$ $\left.\psi_{32}\left(r u_{5}\right)\right|_{A}$ and $V_{46}(r)=\left.\psi_{23}\left(r u_{4}\right)\right|_{A}$ for $r \in F=\mathbb{G}_{a}(F)$.)

Since the torus lies in the image of $\operatorname{Rel}(\mathfrak{C}, \mathfrak{n})$ and $g_{t}^{\dagger}=g_{\sigma_{\mathfrak{n}}(\underline{t})^{-1}}$, the action on $T$ and on the first kind of root groups is the usual $\iota$-action. However,

$$
V_{45}(r)^{\dagger}=\psi_{32}\left(r u_{5}\right)^{\dagger}=\psi_{23}\left(-r u_{4}\right)=V_{46}(-r) \text {. }
$$

So the map $h \mapsto h^{\dagger}$ induces on $S O(A)$ the map $f \mapsto M f M^{-1}$ for

$$
M=\left(\begin{array}{ccc}
1_{4} & & \\
& -S_{2} & \\
& & 1_{4}
\end{array}\right) \text {. }
$$

Write $\eta$ for the 1 -cocycle in $Z^{1}(K / F, O(A))$ given by $\eta_{\iota}=M$. The $K$-map $H \rightarrow O(A)$ descends to a map over $F$ from $H$ onto the twisted group $O(A)_{\eta}$, so we wish to describe the group $O(A)_{\eta}$.

But this is now just a problem of explicitly computing a quadratic form given by descending down a quadratic extension. So we need to find a $K$-basis of $A \otimes K$ consisting of elements fixed by the map $a \otimes \kappa \mapsto M(a) \otimes \iota(\kappa)$. Then $O(A)_{\eta}$ is isomorphic to $O(q)$, where $q$ is the restriction of $N_{A}$ to the $F$-span of those fixed vectors. Such a $K$-basis is given by $u_{i}$ for $1 \leq i \leq 8, e_{1}-e_{2}$, and $\sqrt{k} e_{1}+\sqrt{k} e_{2}$. These vectors give an orthogonal basis for a quadratic form $4 \mathcal{H} \perp\langle-1, k\rangle$, which proves the claim.

Following is a little lemma which foreshadows the way we will prove the Main Theorem for quasi-split groups of type ${ }^{2} E_{6}$.

Lemma 5.4. The Rost multiplier of the inclusion $H^{\circ} \subset E_{6}^{K}$ is 1 . The restriction of the Rost invariant on $H^{1}\left(F, E_{6}^{K}\right)$ to the image of $H^{1}\left(F, H^{\circ}\right)$ has trivial kernel.

Proof. Since the Rost multiplier is invariant under scalar extension, we may work over $K$, where this embedding is described in 5.2. Then some of the coroots (identified with copies of $\mathbb{G}_{m}$ lying in the maximal torus $T$ from 5.3) for $H^{\circ}$ are the same as those for $\operatorname{Spin}(\mathfrak{n})$ considered as a subgroup of $\operatorname{Inv}(J)$ via the map $g$. Since the inclusion $\operatorname{Spin}(\mathfrak{n}) \hookrightarrow \operatorname{Inv}(J)$ has Rost multiplier 1 , so does $H^{\circ} \subset E_{6}^{K}$.

Since the quadratic form $q=4 \mathcal{H} \perp\langle-1, k\rangle$ is isotropic, the spinor norm map $S O(q)(F) \rightarrow F^{*} / F^{* 2}$ is surjective. The Rost invariant $R_{H^{\circ}}$ has trivial kernel by 1.4 , and the second claim follows.

\section{Special cocycles}

Definition 6.1. For $\underline{a}=\left(a_{0}, a_{1}, a_{2}\right) \in\left(F^{*}\right)^{\times 3}$ with product 1 , we define a "special" cocycle $z:=z_{K, \underline{a}}$ in $H^{1}(K / F, G)$. Set $z_{\iota}=\left(z_{0}, z_{1}, z_{2}\right)$ where $z_{j}=m_{j}(\underline{a}) d P$ 
for $P$ the permutation matrix giving the map $u_{k} \mapsto u_{\pi(k)}$ for $\pi$ the permutation $(12)(36)(45)(78), m_{j}(\underline{a})$ the diagonal matrix

$$
m_{j}(\underline{a}):=\operatorname{diag}\left(1, a_{j}, a_{j}, a_{j+2}^{-1}, a_{j+1}^{-1}, 1,1, a_{j}\right)
$$

with subscripts taken modulo 3 , and

$$
d:=\operatorname{diag}(1,1,-1,1,1,-1,1,1) .
$$

The $z_{j}$ form a related triple by $[\mathrm{Gar} 98,1.6,1.7,1.5(3)]$, so $z_{\iota} \in G(K)$. Note that $\sigma_{\mathfrak{n}}\left(m_{j}(\underline{a})\right)=P m_{j}(\underline{a}) P$, and, since $P$ is an isometry of $\mathfrak{n}, \sigma_{\mathfrak{n}}(P)=P^{-1}=P$. We have

$$
{ }^{\iota} z_{j}=\sigma_{\mathfrak{n}}\left(m_{j}(\underline{a}) d P\right)^{-1}=P m_{j}(\underline{a})^{-1} P d P=z_{j}^{-1}
$$

and so $z$ is indeed in $Z^{1}(K / F, G)$.

The image of $z_{K, \underline{a}}$ in $H^{1}(K / F, P)$ is the class of $\underline{a}$.

6.3. Freedom in the definition. Of course, some of these special cocycles are cohomologically equivalent in $H^{1}(K / F, G)$. If $\underline{a}$ and $\underline{a}^{\prime}$ are two triples in $\left(F^{*}\right)^{\times 3}$ such that $a_{j}^{-1} a_{j}^{\prime} \in N_{K / F}\left(K^{*}\right)$ for all $j$, fix $\lambda_{j} \in K^{*}$ such that $a_{j}^{-1} a_{j}^{\prime}=\lambda_{j} \iota\left(\lambda_{j}\right)$. Then for $\underline{\ell}=\left(\ell_{0}, \ell_{1}, \ell_{2}\right)$ with $\ell_{j}=P m_{j}(\underline{\lambda}) P, \underline{\ell}$ is a related triple by [Gar98], so $\underline{\ell} \in G(K)$. We have ${ }^{\iota} \underline{\ell}\left(z_{K, \underline{a}^{\prime}}\right) \iota \underline{\ell}^{-1}=\left(z_{K, \underline{a}}\right)_{\iota}$, i.e., the two cocycles $z_{K, \underline{a}}$ and $z_{K, \underline{a}^{\prime}}$ are cohomologous.

6.4. We will twist by these cocycles to move a cocycle in $H^{1}(F, G)$ so that it takes values in a semisimple group. For now, we just observe that the semisimple group we get from one of them, $\operatorname{Spin}(\mathfrak{n})_{z}$, is described in [Gar98, pp. 403, 404]: Let $k \in F^{*}$ be such that $K=F(\sqrt{k})$ and let $Q_{i}$ denote the quaternion algebra $\left(k, a_{i}\right)_{F}$ generated by elements $x, y$ such that $x^{2}=k, y^{2}=a_{i}$, and $x y=-y x$. The group $\operatorname{Spin}(\mathfrak{n})_{z}$ is isomorphic to $\operatorname{Spin}\left(A_{i}, \sigma_{i}\right)$ where $A_{i}$ is isomorphic to $M_{4}\left(Q_{i}\right), \sigma_{i}$ is an isotropic orthogonal involution with trivial discriminant, and

$$
\left(C_{0}\left(A_{i}, \sigma_{i}\right), \underline{\sigma_{i}}\right) \cong\left(A_{i+1}, \sigma_{i+1}\right) \times\left(A_{i+2}, \sigma_{i+2}\right),
$$

where the subscripts are taken modulo 3. (These properties specify the $\sigma_{i}$ up to isomorphism [Gar01a, 2.3].)

The Moving Lemma lets us say something useful about the Rost invariant of our special cocycles.

Corollary 6.6. The Rost invariant $R_{E_{6}^{K}}\left(z_{K, \underline{a}}\right)$ is trivial if and only if $z_{K, \underline{a}}$ is cohomologically trivial in $H^{1}\left(F, E_{6}^{K}\right)$.

Proof. Consider the element $j=\left(\begin{array}{ccc}0 & 0 & \cdot \\ 0 & 0 & c \\ 0 & 0 & 0\end{array}\right)$ in $e_{0} \times J_{K}$ for $c=u_{2} / 2+u_{8}$. Then $\mathfrak{n}(c)=0$ and, consulting the explicit formula for $j^{\#}$ in [Jac68, p. 358], we see that 
$j^{\#}=0$. Moreover, for $z:=z_{K, \underline{a}}$, we have $z_{\iota} \iota j=\left(\begin{array}{ccc}0 & 0 & \\ 0 & 0 & c^{\prime} \\ 0 & 0 & 0\end{array}\right)$ for $c^{\prime}=u_{1} / 2+u_{7}$. Then

$$
T\left(j, j^{\prime}\right)=c \overline{c^{\prime}}+\bar{c} c^{\prime}=\mathfrak{n}\left(c, c^{\prime}\right)=1
$$

Applying the Moving Lemma shows that $z$ is equivalent in $H^{1}\left(K / F, E_{6}^{K}\right)$ to some $z^{\prime} \in Z^{1}(K / F, G)$ whose image in $H^{1}(K / F, P)$ is $\left(1, a_{0}, a_{0}^{-1}\right)$. In particular, the 0 -component of the triple $z_{\iota}^{\prime}$ in $G O^{\circ}(\mathfrak{C}, \mathfrak{n})^{\times 3}$ belongs to $S O(\mathfrak{C}, \mathfrak{n})$ and the $1-$ and 2-components have multipliers $a_{0}$ and $a_{0}^{-1}$ respectively. Thus the restriction of $z_{\iota}^{\prime}$ to the 10-dimensional subalgebra $A$ defined in 5.2 has determinant 1 and so lies in $H^{\circ}$. If the Rost invariant $R_{E_{6}^{K}}(z)$ is trivial, then $z_{K, \underline{a}}$ is trivial in $H^{1}\left(F, E_{6}^{K}\right)$ by Lemma 5.4 .

In a special case the value of the Rost invariant of our special cocycles can be computed explicitly.

Lemma 6.7. For $a, k \in F^{*}$ such that $K=F(\sqrt{k})$, the Rost invariant of the 1 -cocycle $z_{K,\left(1, a, a^{-1}\right)}$ is $(a) \cup(k) \cup(-1)$ in $H^{3}(F, \mathbb{Z} / 2) \subset H^{3}(F, \mathbb{Q} / \mathbb{Z}(2))$.

Proof. The cocycle $z:=z_{K,\left(1, a, a^{-1}\right)}$ takes values in $H$ and restricts to have determinant 1 on the subalgebra $A$ defined in 5.2 , so $z \in Z^{1}\left(K / F, H^{\circ}\right)$. Since the inclusion $H^{\circ} \subset E_{6}^{K}$ has Rost multiplier 1, to compute the Rost invariant of $z$, we may compute the Rost invariant of $z$ in $H^{1}\left(F, H^{\circ}\right)$. But recall that $H^{\circ}$ is isomorphic to $\operatorname{Spin}(q)$ for $q=4 \mathcal{H} \perp\langle-1, k\rangle$ and that $H^{1}(F, S O(q))$ classifies nondegenerate quadratic forms of the same dimension and discriminant as $q$. So we can compute the Rost invariant of $z$ by computing the quadratic form $q_{z}$ corresponding to the image of $z$ in $H^{1}(F, S O(q))$, which is just the restriction of $\mathfrak{q} \otimes K$ to the vector subspace fixed by the action $a \otimes \kappa \mapsto z_{\iota} M(a) \otimes \iota(\kappa)$ for $M$ as in 5.3.

We perform the Galois descent calculation by decomposing $A \otimes K$ into 2dimensional subspaces and calculating the Galois action on those subspaces.

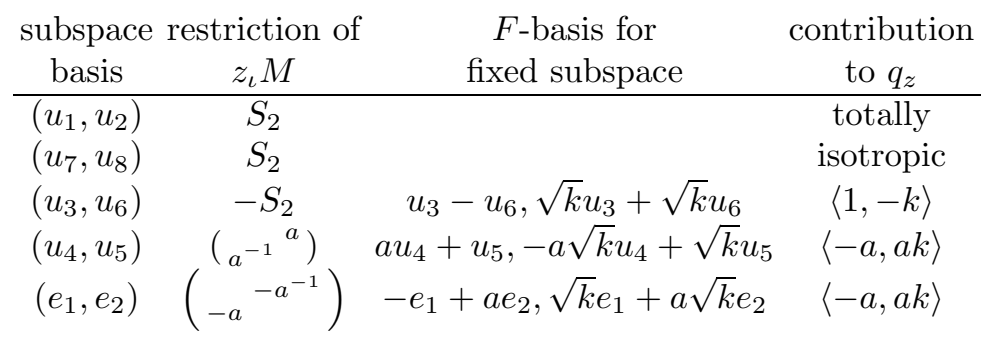

The first two subspaces form a complementary pair of totally isotropic subspaces, so they contribute two hyperbolic planes to $q_{z}$. Thus the image of $z$ is $q_{z}=$ $2 \mathcal{H} \perp\langle 1,-k,-a, a k,-a, a k\rangle$ and the Rost invariant of $z$ is the Arason invariant of $q_{z}-q=\ll a, k,-1 \gg$. 


\section{Quasi-split groups of type $E_{6}$ and $E_{7}$}

This section consists solely of a proof of the main theorem, beginning with a nearly trivial lemma.

Lemma 7.1. Suppose that $C$ is a central subgroup in a simple simply connected group $\Gamma$. Then $H^{1}(F, C)$ acts on $H^{1}(F, \Gamma)$ and for $\zeta \in H^{1}(F, C)$ and $\gamma \in H^{1}(F, \Gamma)$, we have

$$
R_{\Gamma}(\zeta \cdot \gamma)=R_{\Gamma}(\zeta)+R_{\Gamma}(\gamma)
$$

where $R_{\Gamma}(\zeta)$ denotes the image of $\zeta$ under the composition $H^{1}(F, C) \longrightarrow H^{1}(F, \Gamma)$ $\stackrel{R_{\Gamma}}{\longrightarrow} H^{3}(F, \mathbb{Q} / \mathbb{Z}(2))$.

Proof. Pick a 1-cocycle $z \in Z^{1}(F, C)$ which represents $\zeta$. We have a diagram

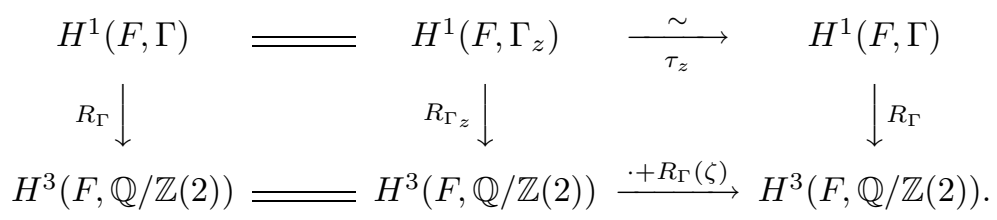

Here the group $\Gamma_{z}$ is the usual twist of $\Gamma$ by the cocycle $z$; it is just the group $\Gamma$ with a different Galois action so that a member $\sigma$ of $\operatorname{Gal}\left(F_{\mathrm{sep}} / F\right)$ maps $g \mapsto z_{\sigma}{ }^{\sigma} g z_{\sigma}^{-1}$. In our case, $z_{\sigma}$ is central, so in fact $\Gamma_{z}$ is identical to $\Gamma$. The map $\tau_{z}$ is the usual twisting map [Ser94, I.5.5], defined by sending $a \in Z^{1}\left(F, \Gamma_{z}\right)$ to the 1-cocycle $\sigma \mapsto a_{\sigma} z_{\sigma}$. The composition of the two maps on the top row is then the action of $\zeta$.

The left-hand box commutes because the Rost invariant is canonical. The righthand box commutes by [Gil00, p. 76, Lem. 7]. The desired equality is equivalent to the commutativity of the outer rectangle.

This result has the obvious corollary that the induced map $H^{1}(F, C) \longrightarrow$ $H^{3}(F, \mathbb{Q} / \mathbb{Z}(2))$ is a group homomorphism.

7.2. Groups of type ${ }^{1} E_{6}$. Suppose first that our simply connected quasisplit group of type $E_{6}$ is split and denote it simply by $E_{6}$. From Example 3.4, we have an embedding $F_{4} \times \boldsymbol{\mu}_{3} \hookrightarrow E_{6}$ which induces a surjection on $H^{1}$ terms. So for $\varepsilon \in H^{1}\left(F, E_{6}\right)$, we can find $\phi \in H^{1}\left(F, F_{4}\right)$ and $\zeta \in H^{1}\left(F, \mu_{3}\right)$ such that $\phi \oplus \zeta \mapsto \varepsilon$. Since $E_{6}$ is split and the image of $\boldsymbol{\mu}_{3}$ is the center of $E_{6}$, the image of $H^{1}\left(F, \boldsymbol{\mu}_{3}\right) \rightarrow H^{1}\left(F, E_{6}\right)$ is trivial. If $\varepsilon$ is in the kernel of the Rost invariant $R_{E_{6}}$, by Lemma $7.1 \phi$ must be killed by the composition

$$
H^{1}\left(F, F_{4}\right) \rightarrow H^{1}\left(F, E_{6}\right) \stackrel{R_{E_{6}}}{\longrightarrow} H^{3}(F, \mathbb{Q} / \mathbb{Z}(2)) .
$$

As described in 2.4, the Rost multiplier of the embedding $F_{4} \subset E_{6}$ is 1 , so $\phi$ lies in the kernel of the Rost invariant $R_{F_{4}}$, which is known to be trivial. Thus $\varepsilon$ is the image of $\zeta$, which we have already observed is trivial. 
Remark 7.3 (Noninjectivity for ${ }^{1} E_{6}$ ). The Rost invariant is typically noninjective for the group $E_{6}$. To see this, we can not simply apply Remark 0.6 and the fact that the embedding $F_{4} \hookrightarrow E_{6}$ has Rost multiplier 1, since two isotopic Albert algebras have the same image in $H^{1}\left(F, E_{6}\right)$.

Instead, fix a ground field $F$ which supports a division (= nonreduced) Albert $F$-algebra $J$. Over the field $F(t)$, the norm $N$ of $J$ does not represent $t$ as can be seen by elementary valuation theory [Jac68, p. 417, Lem. 1]. Consequently, $N$ is not isomorphic to $t N$ over $F(t)$, so the images of the two classes $(J) \oplus(1)$ and $(J) \oplus(t)$ under the map $H^{1}\left(F, F_{4}\right) \times H^{1}\left(F, \boldsymbol{\mu}_{3}\right) \rightarrow H^{1}\left(F, E_{6}\right)$ are distinct by [Gar01b, 2.8(2)]. However, since the image of $H^{1}\left(F, \boldsymbol{\mu}_{3}\right) \rightarrow H^{1}\left(F, E_{6}\right)$ is trivial, by Lemma 7.1 the two classes in $H^{1}\left(F, E_{6}\right)$ have the same Rost invariant.

7.4. Groups of type ${ }^{2} E_{6}$. Suppose now that our quasi-split simply connected group of type $E_{6}$ is not actually split, so that it only becomes split over some quadratic field extension $K$ of $F$. Write $E_{6}^{K}$ for this group, as we have since Section 4. By the split case, any $\alpha \in H^{1}\left(F, E_{6}^{K}\right)$ which is in the kernel of the Rost invariant must become trivial over $K$ and so belongs to $H^{1}\left(K / F, E_{6}^{K}\right)$. Applying Ferrar's Lemma 4.6, we have that $\alpha$ is the image of some $\beta \in H^{1}(K / F, G)$.

7.4. Twisting. Fix a triple $\underline{a}=\left(a_{0}, a_{1}, a_{2}\right) \in\left(F^{*}\right)^{\times 3}$ such that $a_{0} a_{1} a_{2}=1$ which represents the image of $\beta$ in $H^{1}(K / F, P)$. (This makes sense thanks to the description of $H^{1}(K / F, P)$ in 4.7.) Then we set $z:=z_{K, \underline{a}}$ as defined in 6.1 , and we can twist $E_{6}^{K}$ by $z$ to obtain a diagram

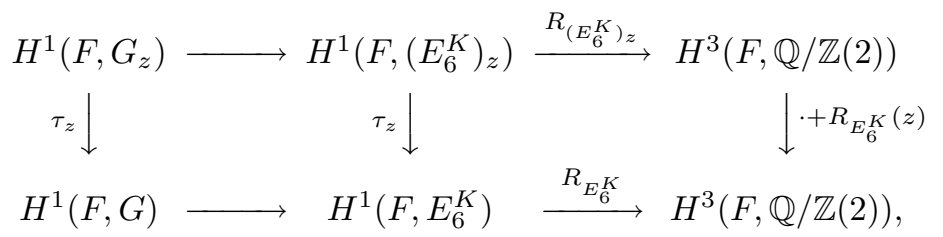

where the right vertical arrow has the specified value by [Gil00, p. 76, Lem. 7].

7.6. The image of $\tau_{z}^{-1}(\beta)$ in $H^{1}(F, S O(A, \sigma))$. We want to say something about what kind of class $\beta^{\prime}:=\tau_{z}^{-1}(\beta)$ can be. In particular, its image in $H^{1}\left(K / F, P_{z}\right)$ is trivial, so $\beta^{\prime}$ comes from the semisimple part of $G_{z}$, which is isomorphic to $\operatorname{Spin}(A, \sigma)$ for $(A, \sigma)$ one of the three algebras $A_{i}$ described in 6.4.

We may think of $\beta^{\prime}$ as lying in $H^{1}(K / F$, Spin $(A, \sigma))$ and consider its image in $H^{1}(K / F, S O(A, \sigma))$. Let $L$ be a generic splitting field of $A$ (e.g., a function field of its Severi-Brauer variety) and consider the image of $\beta^{\prime}$ in $H^{1}(L, S O(A, \sigma))$. Since $A$ is split by $L, \sigma$ becomes adjoint to the quadratic form $\ll k, a_{i+1} \gg \perp 2 \mathcal{H}$ [Gar01a, $2.3]$. The image of $\beta^{\prime}$ determines an 8-dimensional quadratic form $q$ over $L$, and the Rost invariant of $\beta^{\prime}$ is just the class of $q-\ll k, a_{i+1} \gg$ in $I^{3} L / I^{4} L$. However, by the twisting argument above, the Rost invariant of $\beta^{\prime}$ over $F$ is $-R_{E^{K}}(z)$. Since $A$ is split over $L, a_{i} \in L^{*}$ is a norm from $K L$, so by 6.3 and Lemma 6.7 the Rost invariant becomes $(k) \cup\left(a_{i+1}\right) \cup(-1)$ over $L$. 
For $\phi=\ll k, a_{i+1} \gg$, we have that $q-\phi$ lies in $I^{3} L$ and $q-\phi \equiv \phi \ll-1 \gg$ $\bmod I^{4} L$. But then

$$
q+\phi=(q-\phi)+2 \phi \equiv 4 \phi \equiv 0 \bmod I^{4} L .
$$

So $q+\phi$ is in $I^{4} L$. However $\operatorname{dim}(q \perp \phi)=12<16$, so by the Arason-Pfister Hauptsatz, $q \perp \phi$ is hyperbolic and we have $q \cong\langle-1\rangle \phi \perp 2 \mathcal{H}$.

Consequently, the image of $\beta^{\prime}$ in $H^{1}(L, S O(A, \sigma))$ is the same as the image of $-1 \in F^{*} / F^{* 2}=H^{1}(F, Z(S O(A, \sigma)))$. Since $A$ is Brauer-equivalent to a quaternion algebra, it follows from the material in [Sch85, Ch. 10] that the canonical map $H^{1}(F, S O(A, \sigma)) \rightarrow H^{1}(L, S O(A, \sigma))$ is injective. (This was shown independently in [PSS] and [Dej01].) Thus the image of $\beta^{\prime}$ in $H^{1}(F, S O(A, \sigma))$ must also be -1 .

7.7. More generally, any simply connected group $\Gamma$ of type ${ }^{1} D_{4}$ is isomorphic to $\operatorname{Spin}\left(A_{i}, \sigma_{i}\right)$ for three central simple algebras $A_{i}$ of degree 8 with $i=0,1,2$ endowed with an orthogonal involution $\sigma_{i}$ with trivial discriminant and related as in $(6.5)$.

Each of the three descriptions of $\Gamma$ comes paired with natural maps $\Gamma \rightarrow$ $S O\left(A_{i}, \sigma_{i}\right) \rightarrow P \Gamma$ for $P \Gamma$ the adjoint group associated to $\Gamma$. The kernel of the second map is $Z\left(S O\left(A_{i}, \sigma_{i}\right)\right) \cong \boldsymbol{\mu}_{2}$, and the kernel of the composition is $Z(\Gamma)$, which is isomorphic to the subgroup of $\boldsymbol{\mu}_{2}^{\times 3}$ of elements with product 1 . The group $H^{1}(F, Z(\Gamma))$ can be identified with the set of triples $\underline{b}=\left(b_{0}, b_{1}, b_{2}\right) \in$ $F^{*} / F^{* 2}$ with product 1 [KMRT98, 44.14] and where the map $H^{1}(F, Z(\Gamma)) \rightarrow$ $H^{1}\left(F, Z\left(S O\left(A_{i}, \sigma_{i}\right)\right)\right)$ is given by $\underline{b} \mapsto b_{i}$.

Lemma 7.8. (Notation as in the preceding paragraph.) Suppose an element $\eta \in H^{1}(F, \Gamma)$ has the same image in $H^{1}\left(F, S O\left(A_{i}, \sigma_{i}\right)\right)$ as $c_{i} \in F^{*} / F^{* 2}=$ $H^{1}\left(F, Z\left(S O\left(A_{i}, \sigma_{i}\right)\right)\right)$ for $i=1,2$. Then $\eta$ is the image of $\left(\left(c_{1} c_{2}\right)^{-1}, c_{1}, c_{2}\right)$ coming from $H^{1}(F, Z(\Gamma))$.

Proof. The short exact sequence $1 \rightarrow Z\left(S O\left(A_{i}, \sigma_{i}\right)\right) \rightarrow S O\left(A_{i}, \sigma_{i}\right) \rightarrow P \Gamma \rightarrow$ 1 , gives that $\eta$ is killed by the composition $H^{1}(F, \Gamma) \rightarrow H^{1}\left(F, S O\left(A_{i}, \sigma_{i}\right)\right) \rightarrow$ $H^{1}(F, P \Gamma)$ for $i=1$. Thus $\eta$ is the image of some class $\left(n_{0}, n_{1}, n_{2}\right)$ in $H^{1}(F, Z(\Gamma))$.

For general Galois-cohomological reasons, the map $H^{1}(F, Z(\Gamma)) \rightarrow H^{1}(F, \Gamma)$ is a group homomorphism. (Although the second set doesn't have a group structure, the image of the first set does.) The kernel of this map can be described fully by suitably applying [KMRT98, 35.4], but for our purposes it is enough to observe that it contains all elements of the form $\left(s, s^{-1}, 1\right)$ for $s$ a spinor norm of an element in $S O\left(A_{2}, \sigma_{2}\right)(F)$ and symmetrically. Let $G\left(A_{i}, \sigma_{i}\right)^{\circ}$ be the algebraic group of proper similarity factors, i.e., the group with $F$-points

$G\left(A_{i}, \sigma_{i}\right)^{\circ}(F)=\left\{m \in F^{*} \mid \exists f \in A_{i}^{*}\right.$ such that $m=\sigma_{i}(f) f$ and $\left.\operatorname{Nrd}_{A_{i}}(f)=m^{4}\right\}$.

For every $m_{0} \in G\left(A_{0}, \sigma_{0}\right)^{\circ}(F)$, the kernel contains an element of the form $\left(m_{0}, m_{1}, m_{2}\right)$ and symmetrically. Conversely, if $\left(b_{0}, b_{1}, b_{2}\right)$ is in the kernel, then 
$b_{i} \in G\left(A_{i}, \sigma_{i}\right)^{\circ}(F)$ for all $i$.

It is also the case that the natural map $F^{*} / F^{* 2}=H^{1}\left(F, Z\left(S O\left(A_{i}, \sigma_{i}\right)\right)\right) \rightarrow$ $H^{1}\left(F, S O\left(A_{i}, \sigma_{i}\right)\right)$ is a group homomorphism, and its kernel is precisely $G\left(A_{i}, \sigma_{i}\right)^{\circ}(F)$. Thus we may modify $n_{2}$ by an element of $G\left(A_{2}, \sigma_{2}\right)^{\circ}(F)$ and so assume that $n_{2}=c_{2}$.

Now consider the middle component of the triple $\left(n_{0}, n_{1}, n_{2}\right)$. By hypothesis, $n_{1}=m_{1} c_{1}$ for some $m_{1} \in G\left(A_{1}, \sigma_{1}\right)^{\circ}(F)$. By [Mer96, p. 262, Prop.], the group $\operatorname{SN}\left(A_{2}, \sigma_{2}\right)(F)$ of spinor norms from $S O\left(A_{2}, \sigma_{2}\right)(F)$ is the group generated by $F^{* 2}$ and the norms from finite field extensions $E$ which split $A_{2}$ and make $\sigma_{2}$ isotropic. By [Mer96, p. 263, Prop.], $G\left(A_{1}, \sigma_{1}\right)^{\circ}(F)$ is equal to the group generated by the norms from every extension field $E$ which splits $A_{1}$ and makes $\sigma_{1}$ hyperbolic. Since the $\left(A_{i}, \sigma_{i}\right)$ are related by (6.5), any extension which splits $A_{1}$ and makes $\sigma_{1}$ hyperbolic certainly splits $A_{2}$ and makes $\sigma_{2}$ isotropic, so $\operatorname{SN}\left(A_{2}, \sigma_{2}\right)(F) \supseteq$ $G\left(A_{1}, \sigma_{1}\right)^{\circ}(F)$. Consequently, the element $\left(m_{1}, m_{1}^{-1}, 1\right)$ belongs to the kernel of $H^{1}(F, Z(\Gamma)) \rightarrow H^{1}(F, \Gamma)$.

Thus $\eta$ is the image of

$$
\left(n_{0}, n_{1}, n_{2}\right)\left(m_{1}, m_{1}^{-1}, 1\right)=\left(\left(m_{1} c_{1} c_{2}\right)^{-1}, m_{1} c_{1}, c_{2}\right)\left(m_{1}, m_{1}^{-1}, 1\right)=\left(\left(c_{1} c_{2}\right)^{-1}, c_{1}, c_{2}\right)
$$

as desired.

7.9. $\beta^{\prime}$ is in the image of $H^{1}(K / F, Z(\operatorname{Spin}(A, \sigma)))$. Let $(A, \sigma)=\left(A_{0}, \sigma_{0}\right)$ for $\left(A_{i}, \sigma_{i}\right)$ as in 6.4. Combining the result from 7.6 with Lemma 7.8 , we have that $\beta^{\prime} \in H^{1}(F, \operatorname{Spin}(A, \sigma))$ is the image of $(1,-1,-1) \in H^{1}(F, Z(\operatorname{Spin}(A, \sigma)))$. However, for $k \in F^{*}$ such that $K=F(\sqrt{k})$, since $K$ certainly splits $A$ and makes $\sigma$ hyperbolic and $-k=N_{K / F}(\sqrt{k})$, by Merkurjev's norm principle [Mer96, p. 262, Prop.] there is some element of $S O(A, \sigma)(F)$ with spinor norm $-k$. Then as described in the proof of Lemma $7.8, \beta^{\prime}$ is also the image of $\left(1, k, k^{-1}\right) \in$ $H^{1}(F, Z(\operatorname{Spin}(A, \sigma)))$, which itself is in the image of $H^{1}(K / F, Z(\operatorname{Spin}(A, \sigma)))$.

7.10 Consider the 1-cocycle $b=\tau_{z}\left(b^{\prime}\right) \in Z^{1}(K / F, G)$ for $b^{\prime}$ the image of $\left(1, k, k^{-1}\right)$ as above. (Note that $b$ represents the class of $\beta$ and is the 1-cocycle which takes the value $g_{(1,-1,-1)} z_{K, a}$ at $\iota$.) For $j$ and $c$ as in the proof of 6.6 , we set $j^{\prime}:=b_{\iota} \iota j$, so that $j^{\prime}=\left(\begin{array}{ccc}0 & 0 & \dot{c} \\ 0 & 0 & c^{\prime} \\ 0 & 0 & 0\end{array}\right)$ for $c^{\prime}=u_{1} / 2+u_{7}$ and $T\left(j, j^{\prime}\right)=\mathfrak{n}\left(c, c^{\prime}\right)=1$. By the Moving Lemma 4.9 , we may replace $\beta$ by a different inverse image of $\alpha$ in $H^{1}(K / F, G)$ and so assume that $\underline{a}=\left(1, a_{0}, a_{0}^{-1}\right)$.

Any element of $G$ with multiplier $(1, \cdot, \cdot)$ lies in $H$, and since such an element restricts to have determinant 1 on the subspace $A$ defined in 5.2 , it in fact lies in $H^{\circ}$. Thus $\alpha$ is in the image of $H^{1}\left(F, H^{\circ}\right)$. Since the Rost invariant of $\alpha$ is trivial, $\alpha$ must be the trivial class by Lemma 5.4 .

7.11. Groups of type $E_{7}$. We are left with proving that the Rost invariant has trivial kernel for $G$ split of type $E_{7}$, but this follows directly from the same conclusion for quasi-split groups of type $E_{6}$, thanks to Proposition 3.6. 
Acknowledgments. I thank ETH in Zürich for its hospitality while I performed part of the research contained herein; Ph. Gille, Zinovy Reichstein, and Vladimir Chernousov for helpful discussions; and most of all Markus Rost for explaining about his invariants and for posing this problem.

\section{References}

[AH81] B. N. Allison and W. Hein, Isotopes of some nonassociative algebras with involution, J. Algebra 69 (1981), no. 1, 120-142.

[All94] B. N. Allison, Structurable algebras and the construction of simple Lie algebras, Jordan algebras (Berlin) (W. Kaup, K. McCrimmon, and H. P. Petersson, eds.), de Gruyter, 1994, (Proceedings of a conference at Oberwolfach, 1992).

[Bor91] A. Borel, Linear algebraic groups, second ed., Graduate Texts in Mathematics, vol. 126, Springer-Verlag, New York, 1991.

[Bou68] N. Bourbaki, Groupes et algèbres de Lie. Ch. IV, V, VI, Hermann, Paris, 1968, Actualités Scientifiques et Industrielles, No. 1337.

[Bro69] R. B. Brown, Groups of type E $E_{7}, J$. Reine Angew. Math. 236 (1969), 79-102.

[Che00] V. I. Chernousov, private letter, October 2000.

[Dej01] I. Dejaiffe, Formes antihermitiennes devenant hyperboliques sur un corps de déploiement, C. R. Acad. Sci. Paris Sér. I Math. 332 (2001), 105-108.

[EKLV98] H. Esnault, B. Kahn, M. Levine and E. Viehweg, The Arason invariant and mod 2 algebraic cycles, J. Amer. Math. Soc. 11 (1998), no. 1, 73-118.

[Èla72] A. G. Èlašvili, Canonical form and stationary subalgebras of points in general position for simple linear Lie groups, Functional Anal. Appl. 6 (1972), no. 1, 44-53, [Russian original: Funkcional. Anal. i Priložen. 6 (1972), no. 1, 51-62].

[Fer69] J. C. Ferrar, Lie algebras of type $E_{6}$, J. Algebra 13 (1969), 57-72.

[Fer72] J. C. Ferrar, Strictly regular elements in Freudenthal triple systems, Trans. Amer. Math. Soc. 174 (1972), 313-331.

[Fer80] J. C. Ferrar, On the classification of Freudenthal triple systems and Lie algebras of type $E_{7}, J$. Algebra 62 (1980), no. 2, 276-282.

[Gar98] R. S. Garibaldi, Isotropic trialitarian algebraic groups, J. Algebra 210 (1998), 385418, [DOI 10.1006/jabr.1998.7584].

[Gar01a] R. S. Garibaldi, Clifford algebras of hyperbolic involutions, Math. Z. 236 (2001), 321-349, [DOI 10.1007/s002090000180].

[Gar01b] R. S. Garibaldi, Structurable algebras and groups of type $E_{6}$ and $E_{7}, J$. Algebra 236 (2001), no. 2, 651-691, [DOI 10.1006/jabr.2000.8514].

[Gil] Ph. Gille, An invariant of elements of finite order in semisimple simply connected algebraic groups, to appear in J. Group Theory.

[Gil00] Ph. Gille, Invariants cohomologiques de Rost en caractéristique positive, $K$-Theory 21 (2000), 57-100.

[Gil01] Ph. Gille, Cohomologie galoisienne des groupes algebriques quasi-déployés sur des corps de dimension cohomologique $\leq 2$, Compositio Math. 125 (2001), 283-325.

[GV78] V. Gatti and E. Viniberghi, Spinors of 13-dimensional space, Adv. in Math. 30 (1978), no. 2, 137-155.

[Hec84] A. Heck, Involutive automorphisms of root systems, J. Math. Soc. Japan 36 (1984), no. $4,643-658$

[Igu70] J. Igusa, A classification of spinors up to dimension twelve, Amer. J. Math. 92 (1970), 997-1028.

[Jac59] N. Jacobson, Some groups of transformations defined by Jordan algebras. I, J. Reine Angew. Math. 201 (1959), 178-195 (= Oe. 63). 
[Jac61] N. Jacobson, Some groups of transformations defined by Jordan algebras. III, $J$. Reine Angew. Math. 207 (1961), 61-85 (= Oe. 65).

[Jac68] N. Jacobson, Structure and representations of Jordan algebras, AMS Coll. Pub., vol. 39, AMS, Providence, RI, 1968.

[Jac71] N. Jacobson, Exceptional Lie algebras, Lecture notes in pure and applied mathematics, vol. 1, Marcel-Dekker, New York, 1971.

[Kim88] T. Kimura, A classification theory of prehomogeneous vector spaces, Representations of Lie groups (Kyoto, Hiroshima, 1986), Adv. Stud. Pure Math. 14, Academic Press, 1988, 223-256.

[KMRT98] M.-A. Knus and A. S. Merkurjev, M. Rost, and J.-P. Tignol, The book of involutions, Colloquium Publications, vol. 44, American Mathematical Society, Providence, RI, 1998.

[Lam73] T.-Y. Lam, The algebraic theory of quadratic forms, Benjamin, Reading, MA, 1973.

[McC69] K. McCrimmon, The Freudenthal-Springer-Tits constructions of exceptional Jordan algebras, Trans. Amer. Math. Soc. 139 (1969), 495-510.

[Mer96] A. S. Merkurjev, A norm principle for algebraic groups, St. Petersburg Math. J. 7 (1996), no. 2, 243-264.

[MS91] A. S. Merkurjev and A. A. Suslin, The norm residue homomorphism of degree 3, Math. USSR-Izv. 36 (1991), 349-368.

[Pop80] V. L. Popov, A classification of spinors of dimension fourteen, Trans. Mosc. Math. Soc. (1980), no. 1, 181-232, [Russian original: Trudy Moskov. Mat. Obshch. 37 (1978), 173-217].

[Pop94] V. L. Popov, Sections in invariant theory, The Sophus Lie Memorial Conference (Oslo, 1992), Scand. Univ. Press, Oslo, 1994, 315-361.

[PR94] V. P. Platonov and A. Rapinchuk, Algebraic groups and number theory, Academic Press Inc., Boston, MA, 1994.

[PSS] R. Parimala, R. Sridharan, and V. Suresh, Hermitian analogue of a theorem of Springer, J. Algebra, to appear.

[Ros99] M. Rost, On the Galois cohomology of Spin(14), preprint, March 1999.

[Sch66] R. Schafer, An introduction to nonassociative algebras, Pure and Applied Math., vol. 22, Academic Press, 1966.

[Sch69] D. J. Schattschneider, On restricted roots of semi-simple algebraic groups, J. Math. Soc. Japan 21 (1969), 94-115.

[Sch85] W. Scharlau, Quadratic and hermitian forms, Grund. math. Wiss., vol. 270, Springer, Berlin - New York, 1985.

[Ser62] J.-P. Serre, Cohomologie galoisienne des groupes algébriques linéaires, Colloq. Théorie des Groupes Algébriques (Bruxelles, 1962), Librairie Universitaire, Louvain, 1962, 53-68 (= Oe. 53).

[Ser94] J.-P. Serre, Cohomologie galoisienne, fifth ed., Lecture Notes in Mathematics, vol. 5, Springer-Verlag, 1994, [English translation: Galois cohomology, Springer, 1997].

[Ser95] J.-P. Serre, Cohomologie galoisienne: progrès et problèmes, Astérisque (1995), no. 227, 229-257, Séminaire Bourbaki, vol. 1993/94, Exp. 783 (= Oe. 166).

[Sod66] D. Soda, Some groups of type $D_{4}$ defined by Jordan algebras, J. Reine Angew. Math. 223 (1966), 150-163.

[Spr62] T. A. Springer, Characterization of a class of cubic forms, Nederl. Akad. Wetensch. 65 (1962), 259-265.

[SV68] T. A. Springer and F. D. Veldkamp, On Hjelmslev-Moufang planes, Math. Z. 107 (1968), 249-263. 
Ryan Skip Garibaldi

UCLA

Dept. of Mathematics

Los Angeles, CA 90095-1555

USA

e-mail:skip@member.ams.org

(Received: November 1, 2000)

(1) To access this journal online:

4D) http://www.birkhauser.ch 